\title{
SOME CONTRIBUTIONS TO THE THEORY OF RINGS OF OPERATORS ${ }^{(1)}$
}

\author{
BY \\ ERNEST L. GRIFFIN, JR.
}

\section{PREFACE}

The extension of von Neumann's work on factors to general rings of operators on Hilbert spaces of arbitrary dimension has been begun by Dixmier and Kaplansky in [1] and [3] (the numbers in brackets refer to the List of References at the end of the paper). It is the purpose of this paper to extend these results still further, in particular Chapter X of [5] and Chapters I to III of [8].

The general scheme of this paper is as follows: First, the constant $C$ of von Neumann (Chapter $\mathrm{X}$ of [5]) is extended to an operator belonging to the center of a ring of semifinite type, such a ring being one with no type III part. Next, using techniques devised by Dye and von Neumann, this operator $C$ (termed the coupling operator) is shown to be the chief invariant governing the spatial type of a ring. Finally, these results are applied to questions of topology in rings, yielding the fact that the strongest topology is purely algebraic along with the notion of semifinite subring. Besides these main results, we obtain various subsidiary results, in particular, conditions for strong and weak continuity of *-isomorphisms, continuity of the trace in various topologies, and conjugate isomorphism of a ring with its commutant.

The notation of this paper is essentially that of [5] and [7], with but a few exceptions. Throughout the paper, the notation $[M x]$ will denote the closure in some Hilbert space $H$ of the family of vectors $\{A x\}$ for $A$ in a ring $M$ and $x$ a fixed vector. The symbol $\prec$ between projections $E$ and $F(E \prec F)$ in a ring will denote the fact that $E$ is equivalent to a subprojection of $F$ belonging to $M$. This will denote a proper projection only when specifically stated. This particular notation is used because the printer does not have the symbol used in [5] for this relationship. The symbols $\cup$ and $\cap$ will be used in their usual sense of set theoretic union and intersection, the remaining ones being those standard in Hilbert space theory.

In preparing this paper, we have received much valuable assistance from Professors I. E. Segal, I. Kaplansky, and P. R. Halmos, which we gratefully acknowledge.

Note: Since this paper was written, Professor Segal has informed me that

Presented to the Society, April 26, 1952, under the title On semifinite rings; received by the editors October 10, 1952.

(1) Some of the work on this paper was done while the author was an A.E.C. Predoctoral Fellow at the University of Chicago. 
my term "semifinite" is identical with his term "substantial." My attention has further been called to two papers by Robert Pallu de La Barriere; Algèbres auto-adjointes faiblement fermées et algèbres hilbertiennes de classe finie, C. R. Acad. Sci. Paris vol. 232, p. 1994, Isomorphism des *algèbres faiblement fermées d'opérateurs, C. R. Acad. Sci. Paris vol. 234, p. 795, in which some of my results are announced.

\section{Chapter I. The Coupling operator}

1.1. Introduction. In [5, Section 10], there is introduced a finite real constant $C$ which relates a factor $M$ and its commutant $M^{\prime}$. This constant later turns out to have important properties with respect to unitary equivalence. It is the purpose of this chapter to extend the notion of this invariant $C$ to general rings of operators. In later chapters, it will be shown that quite a number of properties of rings depend on this invariant. As this invariant turns out to be an operator in the center of $M$, it is termed the coupling operator. (For a similar operator, defined only for finite rings, see [3].)

Before getting down to the task of defining $C$ we first prove some lemmas of general usefulness.

LEMMA 1.1.1. Let $M$ be a ring with commutant $M^{\prime}$. If $E, E^{\prime}$ are projections in $M, M^{\prime}$ respectively, such that $E E^{\prime}=0$, then there exists a projection $P$ in the center of $M$ such that $E \leqq P, E^{\prime} \leqq P^{\perp}$.

Proof. Consider $\mathscr{X}=\left[x \mid E^{\prime} M^{\prime} x=0\right]$. For $x \in \mathcal{X} ; A \in M, A^{\prime} \in M^{\prime}: E^{\prime} M^{\prime} A x$ $=A E^{\prime} M^{\prime} x=0$ and $E^{\prime} M^{\prime} A^{\prime} x \subseteq E^{\prime} M^{\prime} x=0$, showing that $A \mathcal{X}$ and $A^{\prime} \mathcal{X} \subseteq \mathcal{X}$. Thus $\mathcal{X} \eta M \cap M^{\prime}$ or $P \mathscr{X} C P$ is a projection in the center of $M$. If $x \in H ; E^{\prime} M^{\prime} E x$ $=E E^{\prime} M^{\prime} x=0$, from $E E^{\prime}=0$. Thus $E H \subseteq \mathcal{X}$.

Now consider $E P$ and $E^{\prime} P$ : If $x$ is arbitrary in $H, E P x=E x$ as $E x \in \mathcal{X}$ $=P H$. Thus $E P=E$. Also $E^{\prime} P=0$ as $E^{\prime} \mathcal{X}=0$.

Definition 1.1. A ring $M$ is said to be countably decomposable if every collection $\left\{E_{\alpha}\right\}(\alpha \in \Gamma)$ of orthogonal projections in $M$ is countable.

LEMMA 1.1.2. Let $\omega$ be a positive linear functional on a ring $M$ such that if $\omega(E)=0$ for a projection $E$ in $M$, then $E=0 . M$ is now countably decomposable.

Proof. Let $E_{\alpha}, \alpha \in \Gamma$, be any orthogonal family of projections in $M$. Let $\varepsilon_{k}$ be the set of all $\alpha$ 's $\in \Gamma$ such that $1 /(k+1)<\omega\left(E_{\alpha}\right) \leqq 1 / k$ where $k$ is a positive integer. (Assume that $\omega(I)=1$.) Clearly $\Gamma=\bigcup_{k=1}^{\infty} \varepsilon_{k}$.

However, $\varepsilon_{k}$ contains at most $k \alpha$ 's since if it could contain $k+1 \alpha$ 's, say $\alpha_{1}, \cdots, \alpha_{k+1}$, then $\sum_{i=1}^{k+1} \omega\left(E_{\alpha_{i}}\right)>(k+1) /(k+1)=1$, which is impossible as $\sum_{i=1}^{k+1} \omega\left(E_{\alpha_{i}}\right)=\omega\left(\sum_{i=1}^{k+1} E_{\alpha_{i}}\right) \leqq \omega(I)=1$. Thus, $\Gamma$ is a countable union of sets of finite cardinality, and therefore is countable itself.

Definition 1.2. A positive linear functional $\omega$ on a ring $M$ is termed countably additive if for any countable collection $E_{n}$ of orthogonal projections 
in $M$, we have $\omega\left(\sum_{n=1}^{\infty} E_{n}\right)=\sum_{n=1}^{\infty} \omega\left(E_{n}\right)$. If there exists an $x$ in $H$, a projection $E$ in $M$ such that $\omega\left(A^{*} A\right) \leqq\|A x\|^{2}$ for all $A$ in $M$ with $A E=E A=A$, then we write $\omega \ll(E, x)$ (and also $\omega \ll E)$. If $\omega(A)=(A x, x)$ for all $A$ in $M$ such that $A E=E A=A$, then $\omega \sim(E, x)$ (and also $\omega \sim E$ ).

Note: $\omega \ll E$ is equivalent to the condition obtained from the above by restricting the $A$ 's to projections. The condition then reads: $\omega(F) \leqq\|F x\|^{2}$ for all $F$ in $M$ such that $F \leqq E$. To show this, let $\sum_{k} \alpha_{k} E_{k}\left(\alpha_{k} \geqq 0, E_{k}\right.$ projections in $M$ contained in $E)$ be an approximating sum for $A^{*} A\left(0 \leqq A^{*} A-\sum_{k} \alpha_{k} E_{k}\right.$ $\leqq \epsilon \cdot I)$ and assume $\omega(I)=1$. Then $\omega\left(A^{*} A\right) \leqq \omega\left(\sum_{k} \alpha_{k} E_{k}\right)+\epsilon \leqq \sum_{k} \alpha_{k} \omega\left(E_{k}\right)$ $+\epsilon \leqq \sum_{k} \alpha_{k}\left(E_{k} x, x\right)+\epsilon \leqq\left(\left(\sum \alpha_{k} E_{k}\right) x, x\right)+\epsilon \leqq\left(A^{*} A x, x\right)+\epsilon \leqq\|A x\|^{2}+\epsilon$. Thus we get the inequality $\omega\left(A^{*} A\right) \leqq\|A x\|^{2}+\epsilon$. As $\epsilon$ is arbitrary, we obtain the condition $\omega\left(A^{*} A\right) \leqq\|A x\|^{2}$.

Lemma 1.1.3. Let $\omega$ be a countably additive, positive linear functional on a countably decomposable ring $M$. If for $x$ in $H, P$ a projection of $M, \omega(P) \leqq\|P x\|^{2}$, then there exists a projection $E$ in $M$ such that $0 \neq E \leqq P$ and $\omega \ll(E, E x)$.

Proof. If $\omega \ll P$ is false, there exists, by the preceding note, a projection $E_{0} \leqq P$ such that $\omega\left(E_{0}\right)>\left(E_{0} x, x\right)$. Now using Zorn's Lemma we obtain a maximal collection of orthogonal projections $E_{\alpha}$, such that $E_{\alpha} \leqq P$ and $\omega\left(E_{\alpha}\right)>\left(E_{\alpha} x, x\right)$. As $M$ is countably decomposable, we may index the $E$ 's by integers as $\left\{E_{n}\right\}$. Let $F=\sum_{n=1}^{\infty} E_{n}$.

By countable additivity of $\omega$, we get: $\omega\left(\sum_{n=1}^{\infty} E_{n}\right)=\sum_{n=1}^{\infty} \omega\left(E_{n}\right)$ $>\sum_{n=1}^{\infty}\left(E_{n} x, x\right)=\left(\sum_{n=1}^{\infty} E_{n} x, x\right)$. But as $F \leqq P, \omega(P)=(P x, x)$, and $\omega(F)$ $>(F x, x)$, we cannot have $F=P$. Thus $E=P-F \neq 0$. But now we have $\omega \ll E$; for if $E_{0}$ is a projection in $M$ such that $E_{0} \leqq E$ with the property $\omega\left(E_{0}\right)$ $>\left(E_{0} x, x\right)$, we could add the projection $E_{0}$ to our maximal collection $\left\{E_{\alpha}\right\}$.

LеммA 1.1.4. Let $\omega$ be a positive linear functional on the ring $M$. If $\omega \ll\left(I, z^{\prime}\right)$, then there exists a vector $z$ in $\left[M^{\prime}\right] \cap\left[M^{\prime} z^{\prime}\right]$ such that $\omega \sim(I, z)$.

Proof. Consider the following semilinear form on $M z^{\prime},\left[A z^{\prime}, B z^{\prime}\right]=\omega\left(B^{*} A\right)$ for $A, B$ in $M$. It has the requisite linearity properties and by the Schwarz inequality plus $\omega \ll\left(I, z^{\prime}\right):\left|\left[A z^{\prime}, B z^{\prime}\right]\right|=\left|\omega\left(B^{*} A\right)\right| \leqq\left(\omega\left(A^{*} A\right)\right)^{1 / 2}\left(\omega\left(B^{*} B\right)\right)^{1 / 2}$ $\leqq\left\|A z^{\prime}\right\|\left\|B z^{\prime}\right\|$. This inequality clearly makes it possible to extend the form to $\left[M z^{\prime}\right]$. It is extended to $\left[M z^{\prime}\right]^{\perp}$ by defining $[x, y]=0$ if either $x$ or $y$ is in $\left[M z^{\prime}\right]^{\perp}$. Now by linearity we get the form extended to $H$ with the inequality $[[x, y] \mid \leqq\|x\| \cdot\|y\|$.

For each vector $y$ we now have the linear functional $[x, y]$ on $H$. By continuity and the Riesz Lemma there exists a unique vector $y^{*}$ such that $[x, y]=\left(x, y^{*}\right)$, with $\left(y^{*}, y^{*}\right)=\left\|y^{*}\right\|^{2}=\left[y^{*}, y\right] \leqq\|y\| \cdot\left\|y^{*}\right\|$ and hence $\left\|y^{*}\right\|$ $\leqq\|y\|$ everywhere. Since the map $y \rightarrow y^{*}$ is clearly linear we get a bounded operator $T^{\prime}$ on $H$ such that $T^{\prime} y=y^{*}$.

From the above inequalities, $\left(x, T^{\prime} x\right)=[x, x] \geqq 0$, showing that $T^{\prime} \geqq 0$. We now wish to show that $T^{\prime} \in M^{\prime}$. For $S, A, B$ in $M,\left(A z^{\prime}, T^{\prime} S B z^{\prime}\right)$ 
$=\left[A z^{\prime}, S B z^{\prime}\right]=\omega\left(B^{*} S^{*} A\right)=\left[S^{*} A z^{\prime}, B z^{\prime}\right]=\left(S^{*} A z^{\prime}, T^{\prime} B z^{\prime}\right)=\left(A z^{\prime}, S T^{\prime} B z^{\prime}\right)$ or $\left(A z^{\prime},\left(T^{\prime} S\right) B z^{\prime}\right)=\left(A z^{\prime},\left(S T^{\prime}\right) B z^{\prime}\right)$. By continuity $\left(x, T^{\prime} S y\right)=\left(x, S T^{\prime} y\right)$ for $x$ and $y$ in $\left[M z^{\prime}\right]$. For $y \in\left[M z^{\prime}\right]^{\perp},\left(x, T^{\prime} y\right)=[x, y]=0$ for all $x$, showing that $T^{\prime}$ is zero on $\left[M z^{\prime}\right]^{\perp}$. Now for $x$ and $y$ arbitrary in $H$, let $x=x_{1}+x_{2}$ and $y=y_{1}+y_{2}$ be the decomposition of these vectors into components in $\left[M^{\prime}\right]$ and $\left[M z^{\prime}\right]^{\perp}$ respectively. Then $\left(x, T^{\prime} S y\right)=\left(x_{1}, T^{\prime} S y_{1}\right)+\left(x_{1}, T^{\prime} S y_{2}\right)+\left(x_{2}, T^{\prime} S y\right)$. But $\left(x_{2}, T^{\prime} S y\right)=\left(T^{\prime} x_{2}, S y\right)=0=\left(x_{1}, T^{\prime} S y_{2}\right)$ as $T^{\prime}$ is 0 on $\left[M z^{\prime}\right]^{\perp}$, and $\left[M z^{\prime}\right]^{\perp}$ is invariant under $M$. Thus $\left(x, T^{\prime} S y\right)=\left(x_{1}, T^{\prime} S y_{1}\right)=\left(x_{1}, S T^{\prime} y_{1}\right)$ which in turn equals $\left(x, S T^{\prime} y\right)$ by a computation similar to the above. This proves that $T^{\prime}$ is in $M^{\prime}$. As $T^{\prime} \geqq 0$ we may form $\left(T^{\prime}\right)^{1 / 2}$, which also is in $M^{\prime}$. Let $z=\left(T^{\prime}\right)^{1 / 2} z^{\prime}$.

By the definition of $T^{\prime}, \omega(A)=\left[A z^{\prime}, z^{\prime}\right]=\left(A z^{\prime}, T^{\prime} z^{\prime}\right)=\left(A\left(T^{\prime}\right)^{1 / 2} z^{\prime},\left(T^{\prime}\right)^{1 / 2} z^{\prime}\right)$ $=(A z, z) . z$ is clearly in $\left[M^{\prime} z^{\prime}\right]$ and also is in $\left[M z^{\prime}\right]$, since if $y$ in $\left[M z^{\prime}\right]^{\perp}$, then $|(z, y)|=\left|\left(\left(T^{\prime}\right)^{1 / 2} z^{\prime}, y\right)\right|=\left|\left(z_{1}, \quad\left(T^{\prime}\right)^{1 / 2} y\right)\right| \leqq\left\|z^{\prime} \mid\right\|\left\|\left(T^{\prime}\right)^{1 / 2} y\right\| \leqq\left\|z^{\prime}\right\| \cdot\left(y, T^{\prime} y\right)^{1 / 2}$ $=[y, y]^{1 / 2} \cdot\left\|z^{\prime}\right\|=0$.

\subsection{The coupling operator for $M$ and $M^{\prime}$ finite.}

Lemma 1.2.1. Let $M$ be a finite ring with a vector $z$ such that $\left[M^{\prime} z\right]=H$. If $\omega$ is the linear functional defined for $A$ in $M$ by $\omega(A)=\left(A^{\mathrm{t}} z, z\right)$, then there exists a vector $y$ such that for $A$ in $M, \omega(A)=(A y, y)$ and such that $\left[M^{\prime} y\right]=H$.

Proof. Let $\omega(E)=0$ for $E$ some projection in $M$. Then we have $\left(E^{\natural} z, z\right)$ $=\left\|\left(E^{\natural}\right)^{1 / 2} z\right\|^{2}=0$, or $\left(E^{\natural}\right)^{1 / 2} z=0$. Thus we get $E^{\natural} z=0$. But by $\left[M^{\prime} z\right]=H$, this means $E^{\natural}=0$, and hence that $E$ is 0 . This property plus Lemma 1.1.2 shows us that $M$ is countably decomposable.

Now, by Zorn's Lemma, we pick a maximal collection $\left\{E_{\alpha}\right\}, \alpha \in \Gamma$, with the properties:

1. The $E_{\alpha}$ are mutually orthogonal projections in $M$.

2. There exist vectors $x_{\alpha}$ such that $E_{\alpha} x_{\alpha}=x_{\alpha}$ for all $\alpha$, and such that $\omega \sim\left(E_{\alpha}, x_{\alpha}\right)$.

3. The manifolds $\left[M x_{\alpha}\right]$ are all orthogonal.

Since $M$ is countably decomposable, we see that the $E_{\alpha}$ 's may be indexed by the positive integers $n=1,2, \cdots$, yielding a collection $\left\{E_{n}\right\},\left\{x_{n}\right\}$. Now $P_{\left[M^{\prime} x_{n}\right]} \leqq E_{n}$ follows from $E_{n} A^{\prime} x_{n}=A^{\prime} E_{n} x_{n}=A^{\prime} x_{n}$ for $A^{\prime} \in M^{\prime}$, and then by $\omega\left(E_{n}-P_{\left[M^{\prime} x_{n}\right]}\right)=\left(\left(E_{n}-P_{\left[M^{\prime} x_{n}\right]}\right) x_{n}, x_{n}\right)=\left(x_{n}-x_{n}, x_{n}\right)=0$ we get

$$
E_{n}=P_{\left[M^{\prime} x_{n}\right]} \text {. }
$$

Let $E_{n}^{\prime}=P_{\left[M x_{n}\right]}, E=\sum_{n=1}^{\infty} E_{n}, E^{\prime}=\sum_{n=1}^{\infty} E_{n}^{\prime}$, and $x^{(n)}=\sum_{k=1}^{n} x_{k}$. From $\left\|x_{n}\right\|^{2}=\left(E_{n} x_{n}, x_{n}\right)=\omega\left(E_{n}\right)$ we get $\sum_{k=1}^{n}\left\|x_{k}\right\|^{2}=\sum_{k=1}^{n} \omega\left(E_{k}\right)=\omega\left(\sum_{k=1}^{n} E_{k}\right) \leqq \omega(I)$ for all integers $n$, showing that there exists a vector $x=\sum_{k=1}^{\infty} x_{k}=\lim _{n=\infty} x^{(n)}$. We now observe that $\left[M^{\prime} x\right] \supseteq\left[M^{\prime} E_{n}^{\prime} x\right]=\left[M^{\prime} x_{n}\right]$ and similarly $[M x]$ $\supseteq\left[M x_{n}\right]$, showing that $P_{\left[M^{\prime} x\right]} \geqq E$ and $P_{[M x]} \geqq E^{\prime}$. But it clearly follows from $E x=\sum_{k=1}^{\infty} E x_{k}=\sum_{k=1}^{\infty} E E_{k} x_{k}=\sum_{k=1}^{\infty} E_{k} x_{k}=\sum_{k=1}^{\infty} x_{k}=x$ that $P_{\left[M^{\prime} x\right]} \leqq E$ which finally yields

$$
E=P_{\left[M^{\prime} x\right]}
$$


Similarly,

$$
E^{\prime}=P_{[M x]} .
$$

The next step is to show that $(I-E)\left(I-E^{\prime}\right)=0$. For, if not, there exists a vector $x^{\prime}$ such that $(I-E)\left(I-E^{\prime}\right) x^{\prime}=x^{\prime}$ and $\omega(I-E)=\left\|x^{\prime}\right\|^{2}$. By the countable additivity of $\omega$ (which follows trivially from [1, p. 256, Theorem 17] it follows from Lemma 1.1.3 that there exists a projection $G$ in $M$ such that $G \leqq I-E$ and $\omega \ll\left(G, G x^{\prime}\right)$. Also we have $(I-E)\left(I-E^{\prime}\right) G x^{\prime}$ $=G(I-E)\left(I-E^{\prime}\right) x^{\prime}=G x^{\prime}$. Now applying Lemma 1.1.4 to $M_{G H}$ we get a vector $x^{\prime \prime}$ lying in the manifold $\left[M_{G H}^{\prime} G x^{\prime}\right] \cap\left[M_{G H} G x^{\prime}\right]=\left[M^{\prime} G x^{\prime}\right] \cap G\left[M G x^{\prime}\right]$ $=G\left[M^{\prime} G x^{\prime}\right] \cap\left[M G x^{\prime}\right]=\left[M^{\prime} G x^{\prime}\right] \cap\left[M G x^{\prime}\right]$, such that $\omega \sim\left(G, x^{\prime \prime}\right)$. Clearly, $G x^{\prime \prime}=x^{\prime \prime}$ and $G \perp E_{n}$ for all $n$. Further, for $A, B$ arbitrary in $M,\left(A x^{\prime \prime}, B x_{n}\right)$ $=\left(A x^{\prime \prime}, B E^{\prime} x_{n}\right)=\left(A E^{\prime} P_{\left[M G x^{\prime}\right]} x^{\prime \prime}, B x_{n}\right)=0$ for all $n$ by the fact that $E^{\prime}\left[M G x^{\prime}\right]$ $=\left[M E^{\prime} G x^{\prime}\right]=\left[M E^{\prime}\left(I-E^{\prime}\right)(I-E) G x^{\prime}\right]=0$. Thus $\left[M x^{\prime \prime}\right] \perp\left[M x_{n}\right]$ for all $n$ which shows that $G$ could be added to our "maximal" collection $\left\{E_{n}\right\}$. This contradiction proves that

$$
(I-E)\left(I-E^{\prime}\right)=0 .
$$

Lemma 1.1.1 now provides us with a central projection $P$ such that $(I-E) \leqq P$ and $\left(I-E^{\prime}\right) \leqq P^{\perp}$. Remembering that $\left[M^{\prime} z\right]=H$, consider $[M P z]$ $=P[M z] \subseteq P H=P E^{\prime} H=P[M x]=[M P x]$. By Lemma 9.3.3, p. 179 of $[5]$ (although this lemma applies apparently only to factors, the proof makes no use of the particular properties of factors), this leads to $\left[M^{\prime} P z\right]=P\left[M^{\prime} z\right]$ $=P H<\left[M^{\prime} P x\right]=P\left[M^{\prime} x\right]=P E H$ or $P<P E \leqq P$. Then by finiteness of $M$, $P E \sim P$ which, combined with $(I-E) \leqq P$, yields

$$
E=I \text {. }
$$

Using the fact that $(A B)^{\natural}=(B A)^{\natural}$ for $A, B$ in $M$ (Property $4 \alpha$ of Theorem 10, p. 249 of [1]) we see that $\omega\left(A E_{n}\right)=\omega\left(A E_{n} E_{n}\right)=\omega\left(E_{n} A E_{n}\right)$ for all $A$ in $M$. Hence, $\omega\left(A \sum_{k=1}^{n} E_{k}\right)=\sum_{k=1}^{n} \omega\left(A E_{k}\right)=\sum_{k=1}^{n} \omega\left(E_{k} A E_{k}\right)=\sum_{k=1}^{n}\left(A x_{k}, x_{k}\right)$. But we have $\left(A x^{(n)}, x^{(n)}\right)=\sum_{l=1}^{n} \sum_{k=1}^{n}\left(A x_{k}, x_{l}\right)=\sum_{k=1}^{n}\left(A x_{k}, x_{k}\right)$ as $\left(A x_{k}, x_{l}\right)$

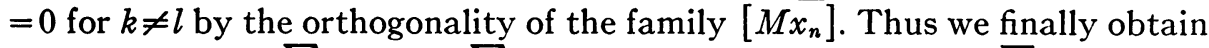
the equation $\omega\left(A \sum_{k=1}^{n} E_{k}\right)=\sum_{k=1}^{n}\left(A x_{k}, x_{k}\right)=\left(A x^{(n)}, x^{(n)}\right)$. As $\sum_{k=1}^{n} E_{k}$ converges strongly to $I$ in the unit sphere of $M$, we have, by the continuity of $\omega, \omega(A)=\lim _{n=\infty} \omega\left(A \sum_{k=1}^{n} E_{k}\right)=\lim _{n=\infty}\left(A x^{(n)}, x^{(n)}\right)=(A x, x)$.

To complete the proof of this lemma, notice that $I=P_{\left[M^{\prime} x\right]}$.

Definition 1.3. A mapping $J$ of $H$ onto itself will be called a conjugation of $H$ if for $x, y$ arbitrary vectors in $H$ and $\alpha$ any complex number we have the following properties: $J(x+y)=J x+J y, J^{2}=I, J \alpha x=\bar{\alpha} J x$, and $(J x, J y)$ $=(y, x)$.

Lemma 1.2.2. Let $M$ be a finite ring. If there exists $a$ vector $z$ such that $[M z]=\left[M^{\prime} z\right]=H$, then there exists a conjugation $J$ of $H$ such that $J M J=M^{\prime}$ and such that for $A$ in the center of $M, J A J=A^{*}$. 
Proof. By Lemma 1.2.1, there exists a vector $x$ such that $\left[M^{\prime} x\right]=H$ and such that $\left(A^{\natural} z, z\right)=(A x, x)=\left(A^{\natural} x, x\right)$ for all $A$ in $M$. Also, by Lemma 9.3.3, p. 179 of $[5],[M x] \sim[M z]=H$; and therefore there is a partial isometry $V^{\prime}$ in $M^{\prime}$ such that $V^{\prime}[M x]=\left[M V^{\prime} x\right]=H$ and $V^{\prime *} V^{\prime} x=x$. But now $\left(A V^{\prime} x, V^{\prime} x\right)$ $=\left(A x, \quad V^{*} V^{\prime} x\right)=(A x, \quad x)=\left(A^{\natural} x, \quad x\right)=\left(A^{\natural} V^{\prime} x, \quad V^{\prime} x\right) \quad$ with $\left[M^{\prime} V^{\prime} x\right]$ $\supseteq\left[M^{\prime} V^{\prime *} V^{\prime} x\right]=\left[M^{\prime} x\right]=H$. Thus if we replace $z$ by $V^{\prime} x$, we get a vector combining the properties of $x$ and $z$, and there is clearly no loss of generality in assuming both properties for our original vector $z$.

We first define $J$ on vectors of the type $A z$ for $A$ in $M$. Here $J A z=A^{*} z$. Clearly we have, for $A$ and $B$ arbitrary operators in $M$,

$$
\begin{aligned}
(J A z, J B z) & =\left(A^{*} z, B^{*} z\right)=\left(B A^{*} z, z\right)=\left(\left(B A^{*}\right)^{\natural} z, z\right)=\left(\left(A^{*} B\right)^{\natural} z, z\right) \\
& =\left(A^{*} B z, z\right)=(B z, A z),
\end{aligned}
$$

yielding

$$
\|J A z\|=\|A z\| .
$$

This last fact shows that $J$ is single-valued since $A z=0$ implies $J A z=0$.

Now since $M z$ is dense in $H$, we can extend $J$ by continuity to all of $H$. Clearly, $(J x, J y)=(y, x)$ for $x$ and $y$ arbitrary in $H$, and $J J A z=J A *^{*} z=A z$ yields $J J=I$. A similar computation yields $J \alpha x=\bar{\alpha} J x$ for $\alpha$ a complex number and $x$ any vector. The additivity of $J$ clearly follows from its additivity on $\mathrm{Mz}$. This completes the proof that $J$ is a conjugation of $H$.

Now for $A$ any bounded operator on $H, J A J$ is clearly additive. That it is linear follows from $J A J \alpha x=J A \bar{\alpha} J x=\alpha J A J x$, for $\alpha$ complex. That it is bounded follows from $\|J A J x\|=\|A J x\| \leqq\|A\|\|J x\|=\|A\|\|x\|$. If we let $A$ be in $M$, with $B, V$ arbitrary in $M$, then

$$
(J A J B) V z=J A V^{*} B^{*} z=B V A^{*} z
$$

and

$$
(B J A J) V z=B J A V^{*} z=B V A^{*} z
$$

showing that $J A J B=B J A J$ on $M z$ and hence on $H$. By arbitrariness of $B$, we find that

\section{$J A J \in M^{\prime}$.}

However, this just shows that $J M J \subseteq M^{\prime}$. A similar computation with $A^{\prime}, B^{\prime}, V^{\prime}$ in $M^{\prime}$ would complete the proof by showing that $J A^{\prime} J \in M$, provided we could prove that $J S^{\prime} z=S^{\prime *} z$ for all $S^{\prime}$ in $M^{\prime}$. To get this result consider: for $B$ arbitrary in $M$ and $S^{\prime}$ fixed in $M^{\prime},\left(J S^{\prime} z, J B^{*} z\right)=\left(B^{*} z, S^{\prime} z\right)=\left(J S^{\prime} z, B z\right)$ $=\left(S^{*} z, B z\right)$ by properties of a conjugation plus the fact that $J B z=B^{*} z$. The above equation now yields $\left(J S^{\prime} z-S^{\prime *} z\right) \perp M z$ and thus $J S^{\prime} z=S^{\prime *} z$. Thus we have proved $J M J=M^{\prime}$.

Now let $A$ be in the center of $M$ with $S$ arbitrary in $M$. Then $(J A J) S z$ 
$=J A S^{*} z=S A^{*} z=A^{*} S z$ as $A^{*}$ is also in the center of $M$. Again by density of $M z$ in $H$, we get $J A J=A^{*}$.

We now prove the following corollary to the above lemma. This result is one of Segal's, namely, Corollary 1.1, p. 9 of [9].

CoRollaRy 1. If $M$ is an abelian ring with a vector $z$ such that $[M z]=H$, then $M$ is maximal abelian $\left(M=M^{\prime}\right)$.

Proof. Since $M$ is abelian, $M$ is finite and $M \subseteq M^{\prime}$. Hence we see that $H=[M z] \subseteq\left[M^{\prime} z\right]$, so that we see that $M$ satisfies the conditions of Lemma 1.2.2. But then the conjugation $J$ satisfies $J M J=M$, and we get finally that $M=M^{\prime}$.

Corollary 2. Let $J$ be a conjugation on $H$ such that $J M J=M^{\prime}$. If $x$ is any vector in $H$, then $J P_{\left[M^{\prime} x\right]} J=P_{[M J x]}$ and $\left(J A J^{*}\right)=J A^{*} J$ for any $A$ in $M$.

Proof. For $x$ and $y$ arbitrary in $H, A$ in $M$, we have: (JAJx,y) $=\left(x,(J A J)^{*} y\right)=(J y, A J x)=\left(A^{*} J y, J x\right)=\left(x, J A^{*} J y\right)$, thus showing that $(J A J)^{*}=J A^{*} J$.

Thus it is clear that $J P_{\left[M^{\prime} x\right]} J$ is a projection in $M^{\prime}$. To get its domain we form $J P_{\left[M^{\prime} x\right]} J H=J P_{\left[M^{\prime} x\right]} H=J\left[M^{\prime} x\right]=J[J M J x]=[M J x]$. This completes the proof of the corollary.

Lemma 1.2.3. Let $M$ be a ring satisfying the conditions of Lemma 1.2.2. If $J$ is the conjugation of that lemma and if $x$ is any vector, then $[M x] \sim[M J x]$ and $\left[M^{\prime} J x\right] \sim\left[M^{\prime} x\right]$.

Proof. By Theorem 6, p. 222 of [1], there exists a projection $P$ in the center of $M$ such that $P[M x]<P[M J x]$ and $P^{\perp}[M x]>P^{\perp}[M J x]$. Since $P$ is in the center of $M$, we get by the previous lemma that $J P J=P$. Hence $P[M J x]=[M P J x]=[M J P x]$ and thus we have $[M P x]<[M J P x]$. Again by Lemma 9.3.3, p. 179 of [5], $\left[M^{\prime} P x\right]<\left[M^{\prime} J P x\right]$; and by using $J M J=M^{\prime}$, one gets a partial isometry $V$ in $M$ such that $V\left[M^{\prime} P x\right]=V J[M J P x]$ $\subseteq\left[M^{\prime} J P x\right]=J[M P x]$. Then by $J J=I$ we get finally that $J V J[M J P x]$ $\subseteq[M P x]$. But $J V J$ is obviously a partial isometry in $M^{\prime}$ and this proves $[M J P x]<[M P x]$. Adding this inequality to our original fact that $[M P x]$ $<[M J P x]$, we obtain the result $[M P x] \sim[M J P x]$. A similar argument for $P^{\perp}$ yields finally $[M x]=[M P x]+\left[M P^{\perp} x\right] \sim[M J P x]+\left[M J P^{\perp} x\right]=[M P J x]$ $+\left[M P^{\perp} J x\right]=[M J x]$. A similar argument proves $\left[M^{\prime} x\right] \sim\left[M^{\prime} J x\right]$.

Lemma 1.2.4. If $\left[M^{\prime} x\right]$ is finite, then $[M x]$ is finite. (See [3, Theorem 1].)

Proof. Let $P_{\left[M^{\prime} x\right]}=E$ and $P_{[M x]}=E^{\prime}$. For an operator $A$ in the ring $M_{E H}$ consider the map $A \rightarrow A E^{\prime}$. This map clearly is from $M_{E H}$ to $M_{E E^{\prime} H}$; and if $A E^{\prime}=0$, then $A x=0$ and hence $A E=0$. But $M_{E H}$ is the set of all operators $B$ in $M$ such that $B E=E B=B$. Thus $A E^{\prime}=0$ implies $A E=0$ and then $A=0$, proving that the above defined map is an isomorphism into. Since $M_{E E^{\prime} H}$ is 
the ring $E M E E^{\prime}$, we see that the above map is onto; or $M_{E H}$ is isomorphic to $M_{E E^{\prime} H}$. A similar argument proves $M_{E^{\prime} H}^{\prime}$ isomorphic to $M_{E E^{\prime} H}^{\prime}$.

By hypothesis we now get $M_{E H}^{\prime}$ finite and hence $M_{E E^{\prime} H}$ is finite. Also, $\left[M_{E E^{\prime} H} x\right]=E E^{\prime} H=\left[M_{E E^{\prime} H} x\right]$, so we may apply Lemma 1.2.2 to get a conjugation $J$ such that $J M_{E E^{\prime} H} J=M_{E E^{\prime} H}^{\prime}$. Clearly we now get that $M_{E E^{\prime} H}^{\prime}$ is finite and thus finally the result that $M_{E^{\prime} H}^{\prime}$ is finite. This completes the proof of the lemma.

Lemma 1.2.5. Let $M$ and its commutant $M^{\prime}$ be finite. If there is a vector $z$ such that $\left[M^{\prime} z\right]=H$, then the operator $C=[M z]^{\natural}$ in the center of $M$ has the property $[M x]^{\natural}=C\left[M^{\prime} x\right]^{\natural}$ for every vector $x$. Furthermore, $C^{-1}$ exists.

Proof. By $\left[M^{\prime} x\right] \subseteq\left[M^{\prime} z\right]=H$ and Lemma 9.3.3, p. 179 of $[5],[M x]$ $\prec[M z]$, and there exists a partial isometry $V^{\prime}$ in $M^{\prime}$ such that $\left[M V^{\prime} x\right]$ $=V^{\prime}[M x] \subseteq[M z]$. Then, since equivalent projections have the same trace, there is clearly no loss of generality in assuming $[M x] \subseteq[M z]$.

Let $E^{\prime}=P_{[M z]}, F^{\prime}=P_{[M x]}$, and $F=P_{\left[M^{\prime} x\right]}$. For $A E^{\prime}$ in $M_{E^{\prime} H}$, consider the map $A E^{\prime} \rightarrow A^{\natural} E^{\prime}$. This is a map into the center of $M_{E^{\prime} H}$ and clearly has all the properties of the trace. Hence if we use $\left(A E^{\prime}\right)^{\circ}$ as the trace in $M_{E^{\prime} H}$, then $\left(A E^{\prime}\right)^{\circ}=A^{\natural} E^{\prime}$.

But $\left[M_{E^{\prime} H} z\right]=[M z]=\left[M_{E^{\prime} H}^{\prime} z\right]$, so by Lemma 1.2.2, there exists a conjugation $J$ of $[M z]$ such that $J M_{[M z]} J=M_{[M z]}^{\prime}$. Then by Lemma 1.2.2, Corollary 2,

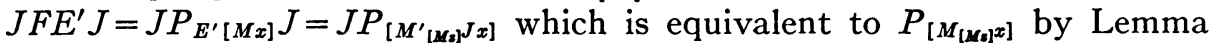
1.2.3. Thus $J F E^{\prime} J \sim P_{\left.\left[M_{[M e]}\right]\right]}=P_{[M x]}=F^{\prime}$. This results in the equation: $J\left(F E^{\prime}\right)^{\circ} J=J F^{\natural} E^{\prime} J=F^{\natural} E^{\prime}=\left(F^{\prime}\right)^{\circ}$, by the continuity properties of $J$ and by $F^{\natural} E^{\prime} \in M_{[M z]} \cap M_{[M z]}^{\prime}$.

To find $\left(F^{\prime}\right)^{\circ}$ we invoke the definition of trace and get a collection $V_{k, r}^{\prime}$, real numbers $a_{k, \tau} \geqq 0$ such that $\sum_{k=1}^{n(\tau)} a_{k, \tau}=1, \tau$ in a directed set $\Gamma$, $n(\tau)$ an integer, $k=1,2, \cdots, n(\tau)$, such that $V_{k, \tau}^{\prime *} V_{k, \tau}^{\prime}=E^{\prime}=V_{k_{r} \tau}^{\prime} V_{k, \tau}^{\prime *}$, $\sum_{k=1}^{n(\tau)} a_{k, \tau} V_{k, \tau}^{\prime *} F^{\prime} V_{k, \tau}^{\prime}$ converges (with respect to $\tau$ ) to $\left(F^{\prime}\right)^{\circ}$ in the uniform topology. But obviously $F^{\prime} \sim$ all $V_{k, \tau}^{\prime *} F^{\prime} V_{k, \tau}^{\prime}$ and therefore, by the uniform continuity of the trace, $\lim _{\tau} \sum_{k=1}^{n(\tau)} a_{k, \tau}\left(F^{\prime}\right)^{\natural}=\left(F^{\prime}\right)^{\natural}=\left(\left(F^{\prime}\right)^{\circ}\right)^{\natural}=F^{\natural}\left(E^{\prime}\right)^{\natural}=C F^{\natural}$. This proves $[M x]^{\natural}=C\left[M^{\prime} x\right]^{\natural}$.

To complete the proof, assume $C x=0$ for some vector $x$. Then $C P_{\left[M^{\prime} x\right]}=0$ $=C P_{\left[M^{\prime} x\right]}^{\natural}=P_{[M x]}^{\natural}$. But this means that $x$ is 0 , and thus $C^{-1}$ exists.

Lemma 1.2.6. Let $M$ be a finite ring with $x$ an arbitrary vector. If $\omega$ is the linear functional defined on $M$ by $\left(A^{\natural} x, x\right)=\omega(A)$, then there exists a projection $P$ in the center of $M$ such that if $E$ is a projection in $M$ with $\omega(E)=0$, then $E \leqq P^{\perp}$.

Proof. Using Zorn's Lemma pick a maximal family of orthogonal projections $\left\{E_{\alpha}\right\}, \alpha \in \Gamma$, in $M$ such that $\omega\left(E_{\alpha}\right)=0$. Let $F=\sum_{\alpha \in \Gamma} E_{\alpha}$. As $F$ is the strong limit in the unit sphere of finite sums of $E_{\alpha}$ 's, we see by additivity and continuity of $\omega$ that $\omega(F)=0$. Again, by Theorem 6, p. 222 of [1], there exists a projection $Q$ in the center of $M$ such that $F Q<F^{\perp} Q$ and $F Q^{\perp}>F^{\perp} Q^{\perp}$. 
This leads to $\omega\left(F^{\perp} Q^{\perp}\right)=\omega\left(\left(F^{\perp}\right)^{\natural} Q^{\perp}\right) \leqq \omega\left((F)^{\natural} Q^{\perp}\right) \leqq \omega\left(F^{\natural}\right)=\omega(F)=0$. But as $F^{\perp} Q^{\perp}$ is a projection orthogonal to $F$, we could add to our "maximal" collection $\left\{E_{\alpha}\right\}, \alpha \in \Gamma$, unless $F^{\perp} Q^{\perp}=0$. Clearly $F Q=0$, and we end up with $F \leqq Q^{\perp}$ and $F^{\perp} \leqq Q$, showing that we must have $F^{\perp}=Q$.

Clearly, the $Q$ above is the $P$ of our lemma; since if $\omega(E)=0$ for some projection $E$ in $M$, then $E \leqq F=Q^{\perp}$, for $E Q \neq 0$ implies that our maximal collection $\left\{E_{\alpha}\right\}$ could be enlarged.

Lemma 1.2.7. Let $M$ be a finite ring. There exists a family $\left\{P_{\alpha}\right\}, \alpha \in \Gamma$, of orthogonal projections in the center of $M$ such that the rings $M P_{\alpha}$ are all countably decomposable, $\sum_{\alpha \in \Gamma} P_{\alpha}=I$.

Proof. By Zorn's Lemma there exists a maximal collection of projections $\left\{P_{\alpha}\right\}, \alpha \in \Gamma$, such that the rings $M P_{\alpha}$ are all countably decomposable and the $\left\{P_{\alpha}\right\}$ are mutually orthogonal. Let $P=\sum_{\alpha \in \Gamma} P_{\alpha}$.

If $P$ is not the identity, pick a nonzero vector $x$ such that $P^{\perp} x=x$. Now, by the previous lemma, there exists a central projection $Q$ with the property that if $\left(E^{\natural} x, x\right)=0$ for some projection $E$ in $M$, then $E \leqq Q^{\perp}$. Then $P x=0$ implies $\left(P^{\natural} x, x\right)=(P x, x)=0$, and finally $P \leqq Q^{\perp}$.

But $M Q$ is countably decomposable by Lemma 1.1 .2 ; so $Q$ may be added to the family $\left\{P_{\alpha}\right\}$. This contradiction proves $P=I$, completing the proof of the lemma.

LEMma 1.2.8. If $M$ is a countably decomposable ring, then there exists a projection $P$ in the center of $M$, vectors $y$ and $z$, such that $[M y]=P H$ and $\left[M^{\prime} z\right]$ $=P^{\perp} H$.

Proof. By Zorn's Lemma there exists a maximal collection of vectors $\left\{x_{\alpha}\right\}$, $\alpha \in \Gamma$, such that the families $\left\{\left[M x_{\alpha}\right]\right\},\left\{\left[M^{\prime} x_{\alpha}\right]\right\}$ consist of orthogonal manifolds. Let $E_{\alpha}=P_{\left[M^{\prime} x_{\alpha}\right]}, E_{\alpha}^{\prime}=P_{\left[M x_{\alpha}\right]}, E=\sum_{\alpha \in \Gamma} E_{\alpha}, E^{\prime}=\sum_{\alpha \in \Gamma} E_{\alpha}^{\prime}$.

If $(I-E)\left(I-E^{\prime}\right) \neq 0$, pick a vector $x^{\prime}$ such that $(I-E)\left(I-E^{\prime}\right) x^{\prime}=x^{\prime} \neq 0$. But it is clear that $\left[M x^{\prime}\right] \perp\left[M x_{\alpha}\right]$ and $\left[M^{\prime} x^{\prime}\right] \perp\left[M^{\prime} x_{\alpha}\right]$ for all $\alpha \in \Gamma$, and hence $x^{\prime}$ could be added to our "maximal" collection $\left\{x_{\alpha}\right\}, \alpha \in \Gamma$. Thus

$$
(I-E)\left(I-E^{\prime}\right)=0 \text {. }
$$

Now, applying Lemma 1.1 .1 , we get a projection $P$ in the center of $M$ such that

and

$$
(I-E) \leqq P
$$

$$
\left(I-E^{\prime}\right) \leqq P_{\perp}
$$

Since $M$ is countably decomposable by hypothesis, the collection $\left\{x_{\alpha}\right\}$ is countable. Hence we may replace $\left\{x_{\alpha}\right\}, \alpha \in \Gamma$, by a sequence $\left\{x_{n}\right\}$. Also, we may assume that $\sum_{n=1}^{\infty}\left\|x_{n}\right\|^{2}<\infty$, for $\left[M x_{n}\right]$ and $\left[M^{\prime} x_{n}\right]$ are not affected by multiplying $x_{n}$ by a constant. 
If $x=\sum_{n=1}^{\infty} x_{n}$ then $\left[M^{\prime} x\right] \supseteq\left[M^{\prime} E_{n}^{\prime} x\right]=\left[M^{\prime} x_{n}\right]$ and $[M x] \supseteq\left[M E_{n} x\right]$ $=\left[M x_{n}\right]$. Thus $P_{[M x]} \geqq E^{\prime}$ and $P_{\left[M^{\prime} x\right]} \geqq E$. But then it is clear that $E x=x$ $=E^{\prime} x$, or $P_{[M x]} \leqq E^{\prime}$ and $P_{\left[M^{\prime} x\right]} \leqq E$, with finally $E=P_{\left[M^{\prime} x\right]}$ and $E^{\prime}=P_{[M x]}$.

To complete the proof of this lemma we observe that: if $y=P x$ and $z=P^{\perp} x$, then

$$
P H=P E^{\prime} H=P[M x]=[M P x]=[M y]
$$

and

$$
P \perp H=P \perp E H=P \perp\left[M^{\prime} x\right]=\left[M^{\prime} P \perp x\right]=\left[M^{\prime} z\right] .
$$

THEOREM 1. Let $M$ and $M^{\prime}$ be finite rings. There exists a unique operator $C \eta M \cap M^{\prime}$ (called the coupling operator for $M, M^{\prime}$ ) such that $[M x]^{\natural}=C\left[M^{\prime} x\right]^{\natural}$ for each vector $x$. Furthermore, $C^{-1}$ exists.

Proof. Using Lemma 1.2.7, we pick orthogonal projections $\left\{P_{\alpha}\right\}, \alpha \in \Gamma$, such that $\sum_{\alpha \in \Gamma} P_{\alpha}=I$ and such that $M P_{\alpha}$ are countably decomposable. Then, by Lemma 1.2.8, one gets projections $E_{\alpha}, \alpha \in \Gamma$, in the center of $M P_{\alpha}, \alpha \in \Gamma$, vectors $x_{\alpha, 1}, x_{\alpha, 2} \in P_{\alpha} H, \alpha \in \Gamma$, such that $\left[M P_{\alpha} x_{\alpha, 1}\right]=E_{\alpha} P_{\alpha} H$ and $\left[M^{\prime} P_{\alpha} x_{\alpha, 2}\right]=E_{\alpha}^{\perp} P_{\alpha} H$.

Now, applying Lemma 1.2.5 to the rings $M_{\left[M^{\prime} x_{\alpha, 2]}\right]}$ and $M_{\left[M_{\alpha, 1}\right]}^{\prime}$, we obtain operators $C_{\alpha}$ and $D_{\alpha}$ in the center of $M$ such that $[M x]^{\natural}=C_{\alpha}\left[M^{\prime} x\right]^{\natural}$ for $x$ in $E_{\alpha}^{\perp} P_{\alpha} H$ and $\left[M^{\prime} x\right]^{\natural}=D_{\alpha}[M x]^{\natural}$ for $x$ in $E_{\alpha} P_{\alpha} H$. Also $C_{\alpha}^{-1}$ and $D_{\alpha}^{-1}$ exist (inverses taken with respect to the $E_{\alpha} P_{\alpha}, E_{\alpha}^{\perp} P_{\alpha}$ ).

Let $C=\sum_{\alpha \in \Gamma}\left(C_{\alpha}+D_{\alpha}^{-1}\right)$. This operator clearly belongs to the center of $M$ and has the obvious inverse $\sum_{\alpha \in \Gamma}\left(C_{\alpha}^{-1}+D_{\alpha}\right)$. For $x$ any vector, we have, by the above equations, that (for all $\alpha$ ) $P_{\alpha} E_{\alpha}^{\perp}[M x]^{\natural}=P_{\alpha} E^{\perp} P_{[M x]}^{\natural}$ $=\left(P_{\alpha} E_{\alpha}^{\perp} P_{[M x]}\right)^{\natural}=P_{\left[M P_{\alpha} E_{\alpha}^{\perp} x\right]}=\left[M P_{\alpha} E_{\alpha}^{\perp} x\right]^{\natural}=C_{\alpha}\left[M^{\prime} P_{\alpha} E_{\alpha}^{\perp} x\right]^{\natural}=C P_{\left[M^{\prime} P_{\alpha} E_{\alpha^{\perp}}{ }^{\natural} x\right]}$ $=C P_{\alpha} E_{\alpha}^{\perp}\left[M^{\prime} x\right]^{\natural}$. Similarly, $P_{\alpha} E_{\alpha}[M x]^{\natural}=C P_{\alpha} E_{\alpha}\left[M^{\prime} x\right]^{\natural}$, and hence $P_{\alpha}[M x]^{\natural}$ $=C P_{\alpha}\left[M^{\prime} x\right]^{\natural}$. Now, by $\sum_{\alpha \in \Gamma} P_{\alpha}=I$, we get finally

$$
[M x]^{\natural}=C\left[M^{\prime} x\right]^{\natural} \text {. }
$$

If $C^{\prime}$ is another operator with the same properties as $C$, consider $\left[M x_{\alpha, 2}\right]^{\natural}$ $=C^{\prime}\left[M^{\prime} x_{\alpha, 2}\right]^{\natural}=C^{\prime} E_{\alpha}^{\perp} P_{\alpha}=C E_{\alpha}^{\perp} P_{\alpha}$ and also $C E_{\alpha} P_{\alpha}=C^{\prime} E_{\alpha} P_{\alpha}$ which yields easily $C=C^{\prime}$. This completes the proof of the theorem.

Definition 1.4. A ring of operators $M$ is said to be semifinite if every nonzero projection in $M$ contains a nonzero finite projection. A ring $M$ is said to be purely infinite if there is no finite nonzero projection in the center of $M$.

1.3. The coupling operator in semifinite rings. In the previous section we introduced the coupling operator for the case where $M$ and $M^{\prime}$ are both finite. It will be the purpose of this section to extend this idea to semifinite rings.

Lemma 1.3.1. Let $M$ be a ring with $E, E^{\prime}$ projections in $M, M^{\prime}$ respectively. If $M$ is semifinite then so are the rings $M^{\prime}, M_{E H}$, and $M_{E^{\prime} H}$. 
Proof. Let $F^{\prime}$ be an arbitrary nonzero projection in $M^{\prime}$. Pick a vector $x$ such that $F^{\prime} x=x \neq 0$ and notice that $P_{[M x]} \leqq F^{\prime}$. Since $M$ is semifinite, the projection $P_{\left[M^{\prime} x\right]}$ contains a nonzero finite projection $G$. Then the manifold $G P_{\left[M^{\prime} x\right]} H=G\left[M^{\prime} x\right]=\left[M^{\prime} G x\right]$ is finite, which implies $[M G x]$ is finite by Lemma 1.2.4. But now $\left[M^{\prime} G x\right] \subseteq\left[M^{\prime} x\right]$ implies by Lemma 9.3 .3 of [5] that $[M G x] \prec[M x]$. Thus there exists a finite manifold belonging to $M^{\prime}$ which is equivalent to the finite manifold $[M G x]$ and contained in $[M x]$. This proves that there is a finite nonzero manifold contained in $F^{\prime}$, and $M^{\prime}$ is shown to be semifinite.

$M_{E H}$ is clearly semifinite as $F(\leqq E)$ is a finite projection in $M_{E H}$ if and only if it is finite in $M$. Further, $M_{E^{\prime} H}=\left(M_{E^{\prime} H}^{\prime}\right)^{\prime}$, so $M_{E^{\prime} H}$ is semifinite.

Lemma 1.3.2. Any semifinite ring $M$ contains a projection $P$ in its center such that $M P$ is finite and $M P^{\perp}$ is purely infinite.

Proof. Using Zorn's Lemma, select a maximal collection $\left\{P_{\alpha}\right\}, \alpha \in \Gamma$, of orthogonal, finite projections in the center of $M$. Clearly, $P=\sum_{\alpha \in \Gamma} P_{\alpha}$ is finite as any partial isometry compressing $P$ also compresses some $P_{\alpha}$. If $Q \neq 0$ is a projection in the center of $M$ such that $Q P=0$, then $Q M$ is infinite, since if not, $Q$ could be added to our maximal collection $\left\{P_{\alpha}\right\}$, $\alpha \in \Gamma$. Thus $M P$ is finite, and $M P^{\perp}$ is purely infinite.

Theorem 2. Let $\left\{E_{\alpha}\right\}, \alpha \in \Gamma,\left\{F_{\alpha}\right\}, \alpha \in \Gamma^{\prime}$, be distinct families of orthogonal, finite, equivalent projections in a purely infinite ring $M$ ( $E_{\alpha}$ not necessarily equivalent to $\left.F_{\gamma}\right)$. If $\sum_{\alpha \in \Gamma} E_{\alpha}=I=\sum_{\gamma \in \Gamma^{\prime}} F_{\gamma}$, then the cardinal of the set $\Gamma$ equals the cardinal of the set $\Gamma^{\prime}$.

Proof. Let $\Omega$ be cardinal of $\Gamma$ and $\Omega^{\prime}$ that of $\Gamma^{\prime}$. Take an arbitrary vector $x$ such that $\|x\|=1$, and consider the functional $(A x, x)=\omega(A)$ on $M \cap M^{\prime}$. By Lemma 1.2.6, there exists a projection $P(\neq 0)$ in the center of $M$ such that $\omega$ is not zero on nonzero projections contained in $P$. Also $P=\sum_{\alpha \in \Gamma} P E_{\alpha}$ $=\sum_{\gamma \in \Gamma^{\prime}} P F_{\gamma}$ with $\left\{P E_{\alpha}\right\},\left\{P F_{\gamma}\right\}$ as families of orthogonal, equivalent, finite projections. Thus, with the aid of the spectral theorem, there is no loss of generality in assuming that there is a linear functional $\omega$ on the center of $M$ such that for $A$ a nonzero, positive semi-definite operator in $M \cap M^{\prime}$, $\omega(A)>0$ and $\omega(I)=1$.

Now let $\varepsilon_{\alpha}^{\prime}=\left[\gamma \in \Gamma^{\prime} \mid E_{\alpha} F_{\gamma} E_{\alpha} \neq 0\right]$. For any $\gamma \in \Gamma^{\prime}$, if $E_{\alpha} F_{\gamma} E_{\alpha}$ $=\left(E_{\alpha} F_{\gamma}\right)\left(\left(E_{\alpha} F_{\gamma}\right)^{*}\right)=0$ for all $\alpha \in \Gamma$, then $E_{\alpha} F_{\gamma}=0$ for all $\alpha \in \Gamma$, which implies $\sum_{\alpha \in \Gamma} E_{\alpha} F_{\gamma}=F_{\gamma}=0$. This contradiction proves that for each $\gamma \in \Gamma^{\prime}$, there exists an $\alpha(\gamma) \in \Gamma$ such that $E_{\alpha(\gamma)} F_{\gamma} E_{\alpha(\gamma)} \neq 0$; but then $\gamma \in \mathcal{E}_{\alpha(\gamma)}$, yielding finally that

$$
\Gamma^{\prime}=\bigcup_{\alpha \in \Gamma} \varepsilon_{\alpha}^{\prime}
$$

As $M_{E_{\alpha} H}$ is finite, we have a trace $\xi_{\alpha}$ in it. Since the center of $M_{E_{\alpha} H}$ is 
$\left(M \cap M^{\prime}\right)_{E_{\alpha} H}$ by Lemma 11.3.2, p. 186 of [5], we may write $\left(E_{\alpha} F_{\gamma} E_{\alpha}\right)^{\natural_{\alpha}}$ $=S_{\gamma}^{\alpha} E_{\alpha}$, where $S_{\gamma}^{\alpha} \in M \cap M^{\prime}$.

Let $\Gamma_{0}^{\prime}$ be an arbitrary finite subset of $\Gamma^{\prime}$. By $\sum_{\gamma \in \Gamma^{\prime}} E_{\alpha} F_{\gamma} E_{\alpha}=E_{\alpha}$ and $E_{\alpha} F_{\gamma} E_{\alpha} \geqq 0,0 \leqq \sum_{\gamma \in \Gamma_{0}{ }^{\prime}}\left(E_{\alpha} F_{\gamma} E_{\alpha}\right)^{\natural_{\alpha}}=\sum_{\gamma \in \Gamma_{0}{ }^{\prime}} S_{\gamma}^{\alpha} E_{\alpha} \leqq E_{\alpha}$. But the $E_{\alpha}$ are all equivalent, and thus for any $\beta \in \Gamma$, there exists a partial isometry $V$ in $M$ such that $V^{*} V=E_{\alpha}$ and $V V^{*}=E_{\beta}$. The above inequality now becomes $0 \leqq \sum_{\gamma \in \mathrm{r}_{0}^{\prime}} S_{\gamma}^{\alpha} V^{*} V \leqq V^{*} V$, and hence $0 \leqq V \sum_{\gamma \in \mathrm{ro}^{\prime}} S_{\gamma}^{\alpha} V^{*} V V^{*} \leqq V V^{*} V V^{*}$. Finally, we get, for all $\beta \in \Gamma$,

$$
0 \leqq \sum_{\gamma \in \Gamma_{0}^{\prime}} S_{\gamma}^{\alpha} E_{\beta} \leqq E_{\beta}
$$

But by hypothesis, $\sum_{\beta \in \Gamma} E_{\beta}=I$, so that we have shown that 0 $\leqq \sum_{\gamma \in \mathbf{r}_{0}^{\prime}} S_{\gamma}^{\alpha} \leqq I$, with $0 \leqq S_{\gamma}^{\alpha}$ in particular. Using our linear functional $\omega, 0 \leqq \sum_{\gamma \in \mathrm{ro}^{\prime}} \omega\left(S_{\gamma}^{\alpha}\right) \leqq 1$, and $\omega\left(S_{\gamma}^{\alpha}\right) \neq 0$ if and only if $S_{\gamma}^{\alpha} \neq 0$. These facts, plus the argument of Lemma 1.1.2, show that the set $\left[\gamma \in \Gamma^{\prime} \mid \omega\left(S_{\gamma}^{\alpha}\right) \neq 0\right]$ must be countable. However, it is clear that $\omega\left(S_{\gamma}^{\alpha}\right) \neq 0$ if and only if $\left(E_{\alpha} F_{\gamma} E_{\alpha}\right)^{\natural_{\alpha}} \neq 0$, and we see that $\varepsilon_{\alpha}^{\prime}=\left[\gamma \in \Gamma^{\prime} \mid\left(S_{\gamma}^{\alpha}\right) \neq 0\right]$ is countable.

Thus $\Omega^{\prime}=$ the cardinal of $U_{\alpha \in \Gamma} \varepsilon_{\alpha}^{\prime} \leqq \boldsymbol{N}_{0} \cdot \Omega=\Omega$. By symmetry, $\Omega \leqq \Omega^{\prime}$ and finally $\Omega=\Omega^{\prime}$.

Definitions 1.5. A semifinite ring $M$ is said to be of homogeneous type if it either satisfies the hypotheses of Theorem 2, or is finite. If the cardinal of Theorem 2 is $\alpha$, then the corresponding homogeneous ring is said to be of type $S_{\alpha}$. If finite, a ring will be said to be of type $S_{1}$.

THEOREM 3. Let $\pi$ be the collection of cardinals containing 1 plus all infinite cardinals $\alpha \leqq$ the cardinal of $H$. If $M$ is a semifinite ring on $H$, then for each $\alpha \in \pi$ there exists a projection $P_{\alpha}$ in the center of $M$ such that the collection $\left\{P_{\alpha}\right\}$ is orthogonal, $\sum_{\alpha \in \pi} P_{\alpha}=I$, and $M P_{\alpha}$ is of type $S_{\alpha}$.

Proof. For $\alpha$ an infinite cardinal in $\pi$, pick, by Zorn's Lemma, a maximal collection $\left\{Q_{\gamma}\right\}, \gamma \in \Gamma$, of orthogonal projections in the center of $M$ such that $\left\{M Q_{\gamma}\right\}, \gamma \in \Gamma$, are of type $S_{\alpha}$. In each ring $M Q_{\gamma}$ we have an orthogonal family $\left\{E_{\gamma, k}\right\}, k \in \kappa$, of equivalent finite projections such that $Q_{\gamma}$ $=\sum_{k \in \kappa} E_{\gamma, k}$. (As cardinals of $\left\{E_{\gamma, k}\right\}$ for each $\gamma \in \Gamma$ are the same, we may choose the same index set $\kappa$ for all $\gamma$.)

Now form $E_{\kappa}=\sum_{\gamma \in \Gamma} E_{\gamma, k}$ for each $k \in \kappa . E_{k}$ is clearly finite as it is the sum of finite projections, each in a different central projection $Q_{\gamma}$, all orthogonal. But now $\sum_{k \in \kappa} E_{k}=\sum_{\mathbf{k} \in \kappa} \sum_{\gamma \in \Gamma} E_{\gamma, k}=\sum_{\gamma \in \Gamma} \sum_{k \in \kappa} E_{\gamma, k}$ $=\sum_{\kappa \in \Gamma} Q_{\gamma}=Q$ shows that $Q M$ is of type $\bar{S}_{\alpha}$, since the $E_{k}$ are clearly equivalent. If $Q^{\prime}$ is any projection in the center of $M$ such that $M Q^{\prime}$ is of type $S_{\alpha}$, then $Q^{\prime} \leqq Q$, since otherwise we could add $Q^{\perp} Q^{\prime}$ to our "maximal" collection $\left\{Q_{\gamma}\right\}, \gamma \in \Gamma$. We have now proved that there exists a maximal projection $Q$ in the center of $M$ such that $M Q$ is of type $S_{\alpha}$. We define $P_{\alpha}=Q$.

We define $P_{1}$ as the $P$ of Lemma 1.3.2 and also $P=\sum_{\alpha \in \pi} P_{\alpha}$. Assume 
$P^{\perp} \neq 0$ and pick a nonzero finite projection $G \leqq P^{\perp}$. By Zorn's Lemma there exists a maximal collection of orthogonal projections $E_{\gamma}, \gamma \in \Gamma$, such that each $E_{\gamma} \sim G$. Let $E=\sum_{\gamma \in \Gamma} E_{\gamma}$, and consider $E^{\perp} P^{\perp}$. Clearly, we cannot have $G \prec E^{\perp} P^{\perp}$, otherwise we could enlarge our maximal collection $\left\{E_{\gamma}\right\}, \gamma \in \Gamma$. Also, if $E^{\perp} P^{\perp}=0$, then $P^{\perp} \leqq E$ which implies $P^{\perp}=E$, as $E$ is the sum of projections equivalent to a projection in $P^{\perp}$. But then $P^{\perp}$ is homogeneous and must lie in $P$, or $P^{\perp}=0$. Thus our assumption of $P^{\perp} \neq 0$ drives us to the possibility that there exists a projection $Q$ in the center of $M$ such that $0 \neq Q E^{\perp} P^{\perp}\left\langle Q G\right.$. Now $Q P^{\perp}=\sum_{\gamma \in \Gamma} Q E_{\gamma}+Q E^{\perp} P^{\perp}$, with the $Q E_{\gamma}$ all equivalent ( $\Gamma$ is clearly infinite, since $P^{\perp} M$ is purely infinite). Assuming the index set $\Gamma$ is well ordered, $Q E_{\gamma} \sim Q E_{\gamma+1}$ and $\sum_{\gamma \geqq 1} Q E_{\gamma} \sim \sum_{\gamma>1} Q E_{\gamma}$. Therefore $\sum_{\gamma \geqq 1} Q E_{\gamma}>\sum_{\gamma>1} Q E+Q E^{\perp} P^{\perp} \sim Q P^{\perp}$, and clearly $Q P^{\perp} \sim \sum_{\gamma \geqq 1} Q E_{\gamma}$. Let $V$ be the partial isometry in $M$ such that $V^{*} V=\sum_{\gamma \geqq 1} Q E_{\gamma}, V V^{*}=P^{\perp} Q$. Then, if $F_{\gamma}=V Q E_{\gamma} V^{*}$, we have the $\left\{F_{\gamma}\right\}$ as a collection of orthogonal, equivalent, finite projections such that $\sum_{\gamma \geqq 1} F_{\gamma}=V V^{*} V V^{*}=V V^{*}=Q P^{\perp}$, showing finally that $Q P^{\perp} M$ is homogeneous. This again contradicts our choice of $P$ and so we have proved that $P=I$.

That the $P_{\alpha}$ are unique is obvious once it is noticed that the $P_{\alpha}$ must be the maximal projection of type $S_{\alpha}$.

Definition 1.6. Let $M$ be a semifinite ring. By Theorem 3, there exists a collection of orthogonal projections $\left\{P_{\alpha}\right\}$ in the center of $M$ such that $M P_{\alpha}$ are of type $S_{\alpha}$, for $\alpha \in \pi$. Using our Lemma 1.3.1, $M^{\prime}$ is semifinite too, and thus there exists a collection $P_{\alpha}^{\prime}, \alpha \in \pi$, such that $M^{\prime} P_{\alpha}^{\prime}$ are of type $S_{\alpha}$, $\sum_{\alpha \in \pi} P_{\alpha}^{\prime}=I$. Now, if $P_{\alpha, \alpha^{\prime}}=P_{\alpha} \cdot P_{\alpha}^{\prime}$, then $\sum_{\alpha, \alpha^{\prime} \in \pi} P_{\alpha, \alpha^{\prime}}=\sum_{\alpha \in \pi} P_{\alpha}$ $\cdot \sum_{\alpha^{\prime} \in \pi} P_{\alpha^{\prime}}^{\prime}=I$, with $M P_{\alpha, \alpha^{\prime}}, M^{\prime} P_{\alpha, \alpha^{\prime}}$ of types $S_{\alpha}, S_{\alpha^{\prime}}$ respectively. We now introduce the operator $C_{1}$, the coupling operator defined in Theorem 1 , for the rings $M P_{1,1}, M^{\prime} P_{1,1}$. We also introduce the formal operators $\left(\alpha / \alpha^{\prime}\right) P_{\alpha, \alpha^{\prime}}$ and form the formal sum

$$
C=C_{1}+\sum_{\left(\alpha, \alpha^{\prime}\right) \neq(1,1)} \frac{\alpha}{\alpha^{\prime}} P_{\alpha, \alpha^{\prime}} .
$$

The formal operator we have just introduced will be called the coupling operator for the rings $M, M^{\prime}$.

It is clear from the above definition that to say that $C$ is the coupling operator for $M, M^{\prime}$ is to give $C_{1}$ as the finite coupling operator plus the fact that $M P_{\alpha, \alpha^{\prime}}, M^{\prime} P_{\alpha, \alpha^{\prime}}$ are of type $S_{\alpha}, S_{\alpha^{\prime}}$ respectively. That such a formal operator is unique follows from the uniqueness of the $\left\{P_{\alpha}\right\}$ and $\left\{P_{\alpha}^{\prime}\right\}, \alpha \in \pi$.

Definition 1.7. Let $\left\{A_{\alpha}\right\}, \alpha \in \Gamma$, be a directed set of bounded operators on a Hilbert space $H$. The above collection is said to converge to 0 in the $\sigma$-weak topology if for every two sets $\left\{x_{n}\right\},\left\{y_{n}\right\} \subseteq H, n$ an integer, such that $\sum_{n=1}^{\infty}\left\|x_{n}\right\|^{2}<\infty, \sum_{n=1}^{\infty}\left\|y_{n}\right\|^{2}<\infty$, then $\sum_{n=1}^{\infty}\left(A_{\alpha} x_{n}, y_{n}\right)$ converges to 0 .

TheOREM 4. Let $M$ be any ring on a Hilbert space $H$ and $\Gamma$ an abstract set 
of cardinal $\Omega$. There exists a ring of operators $\tilde{M}$ on a Hilbert space $\tilde{H}$ with the following properties:

1. There exists a collection $\left\{e_{\gamma}\right\}, \gamma \in \Gamma$, of orthogonal equivalent projections in $\tilde{M}$ such that $\sum_{\gamma \in \Gamma} e_{\gamma}=\tilde{I}$, the identity of $\tilde{M}$, and such that $\tilde{M}_{e_{\gamma} \widetilde{H}}$ are each unitarily equivalent to $M$.

2. There is $a^{*}$-isomorphism $\phi$ of $M^{\prime}$ onto $\tilde{M}^{\prime}$ which is continuous in the strongest and $\sigma$-weak topologies. The inverse map $\phi^{-1}$ is continuous in the weak, strong, strongest, and $\sigma$-weak topologies.

Proof. For each $\gamma \in \Gamma$ choose a replica $H_{\gamma}$ of $H$ and form $\widetilde{H}=\sum_{\gamma \in \Gamma} \oplus H_{\gamma}$, in the standard way. Let $V_{\gamma}$ be the isometry mapping $H$ onto $H_{\gamma}$.

Let $e_{\gamma}=P_{H_{\gamma}}$ and define $e_{\gamma, \gamma^{\prime}}=V_{\gamma^{\prime}} V_{\gamma}^{-1} e_{\gamma}$ for $\gamma \in \Gamma$. Each bounded operator $\tilde{A}$ on $\tilde{H}$ is obviously determined by the collection $e_{\gamma} \tilde{A} e_{\gamma^{\prime}}, \gamma, \gamma^{\prime} \in \Gamma$. We now notice that for $\tilde{A}$ bounded on $\tilde{H}$, the $\theta_{\gamma, \gamma^{\prime}}(\tilde{A})=V_{\gamma}^{-1} e_{\gamma} \tilde{A} e_{\gamma^{\prime}}, V_{\gamma^{\prime}}$ are bounded operators on $H$.

We define $\tilde{M}$ to be all bounded operators on $\widetilde{H}$ such that $\theta_{\gamma, \gamma^{\prime}}(\tilde{A})$ lies in $M$ for all $\gamma, \gamma^{\prime} \in \Gamma$. If $\tilde{A}^{\prime} \in \tilde{M}^{\prime}$, then $e_{\gamma} \tilde{A}^{\prime}=\tilde{A}^{\prime} e_{\gamma}$ for all $\gamma$, and hence by $e_{\gamma} e_{\gamma^{\prime}}=0$ for $\gamma \neq \gamma^{\prime}, e_{\gamma} \tilde{A}^{\prime} e_{\gamma^{\prime}}=\tilde{A}^{\prime} e_{\gamma} e_{\gamma^{\prime}}=0$ for $\gamma \neq \gamma^{\prime}$. From $e_{\gamma, \gamma^{\prime}} \in \tilde{M}$ comes $V_{\gamma^{\prime}} V_{\gamma}^{-1} e_{\gamma} \tilde{A}^{\prime}$ $=\tilde{A}^{\prime} V_{\gamma^{\prime}}, V_{\gamma}^{-1} e_{\gamma}=V_{\gamma^{\prime}} V_{\gamma}^{-1} e_{\gamma} \tilde{A}^{\prime} e_{\gamma}=e_{\gamma^{\prime}} \tilde{A}^{\prime} e_{\gamma^{\prime}}, V_{\gamma^{\prime}} V_{\gamma}^{-1}$ and finally: $\theta_{\gamma, \gamma^{\prime}}\left(\widetilde{A}^{\prime}\right)$ $=V_{\gamma}^{-1} e_{\gamma} \tilde{A}^{\prime} e_{\gamma} V_{\gamma}=V_{\gamma^{\prime}}^{-1} e_{\gamma^{\prime}} \tilde{A}^{\prime} e_{\gamma^{\prime}} V_{\gamma^{\prime}}=\theta_{\gamma^{\prime}, \gamma^{\prime}}\left(\tilde{A}^{\prime}\right)$, for all $\gamma, \gamma^{\prime} \in \Gamma$. Thus it is clear that if $\theta_{\gamma^{\prime}, \gamma^{\prime}}\left(\tilde{A}^{\prime}\right)=0$ for some $\gamma^{\prime} \in \Gamma$, then $e_{\gamma} \tilde{A}^{\prime} e_{\gamma}$ is 0 for all $\gamma \in \Gamma$ or $\tilde{A}^{\prime}=0$. Furthermore, we have, for $\widetilde{B}$ any operator in either $\tilde{M}$ or $\tilde{M}^{\prime}$, for all $\gamma \in \Gamma$, $\theta_{\gamma, \gamma}\left(\tilde{A}^{\prime} \tilde{B}\right)=V_{\gamma}^{-1} e_{\gamma} \tilde{A}^{\prime} \widetilde{B} e_{\gamma} V_{\gamma}=V_{\gamma}^{-1} e_{\gamma} \tilde{A}^{\prime} e_{\gamma} \widetilde{B}_{\gamma} e_{\gamma} V_{\gamma}=V_{\gamma}^{-1} e_{\gamma} \tilde{A}^{\prime} e_{\gamma} V_{\gamma} V_{\gamma}^{-1} e_{\gamma} \widetilde{B} e_{\gamma} V_{\gamma}$ $=\theta_{\gamma, \gamma}\left(\tilde{A}^{\prime}\right) \theta_{\gamma, \gamma}(\tilde{B})$, and hence $\theta_{\gamma, \gamma}$ is an isomorphism of $\tilde{M}^{\prime}$ into the algebra of bounded operators on $H$. Also if $B$ is arbitrary in $M$, consider the operator $V_{\gamma} B V_{\gamma}^{-1} e_{\gamma}$ on $\tilde{H}$. This operator is obviously an operator $\widetilde{B}$ in $\tilde{M}$, and $\theta_{\gamma, \gamma}\left(\widetilde{A}^{\prime}\right) B$ $=\theta_{\gamma, \gamma}\left(\tilde{A}^{\prime}\right) \cdot \theta_{\gamma, \gamma}(\tilde{B})=\theta_{\gamma, \gamma}\left(\tilde{A}^{\prime} \tilde{B}\right)=\theta_{\gamma, \gamma}\left(\tilde{B} \tilde{A}^{\prime}\right)=\theta_{\gamma, \gamma}(\tilde{B}) \cdot \theta_{\gamma, \gamma}\left(\tilde{A}^{\prime}\right)=B \theta_{\gamma, \gamma}\left(\tilde{A}^{\prime}\right)$ proving that $\theta_{\gamma, \gamma}\left(\tilde{A}^{\prime}\right)$ lies in $M^{\prime}$. Conversely, if $B^{\prime}$ is arbitrary in $M^{\prime}$, form $\tilde{B}^{\prime}$ $=\sum_{\delta \in \Gamma} e_{\delta} V_{\delta} B^{\prime} V_{\delta}^{-1} e_{\delta}$. This is a bounded operator on $\tilde{H}$; and if $\tilde{A}$ is arbitrary in $\tilde{M}$, then $\theta_{\gamma, \gamma^{\prime}}\left(\tilde{A} \widetilde{B}^{\prime}\right)=V_{\gamma}^{-1} e_{\gamma} \tilde{A} \tilde{B}^{\prime} e_{\gamma^{\prime}} V_{\gamma^{\prime}}=V_{\gamma}^{-1} e_{\gamma} \tilde{A} e_{\gamma^{\prime}} V_{\gamma^{\prime}} \tilde{B}^{\prime} V_{\gamma^{\prime}}^{-1} e_{\gamma^{\prime}} V_{\gamma^{\prime}}$ $=\theta_{\gamma, \gamma^{\prime}}(\tilde{A}) B^{\prime}=B^{\prime} \theta_{\gamma, \gamma^{\prime}}(\tilde{A})$ which equals $\theta_{\gamma, \gamma^{\prime}}\left(\widetilde{B}^{\prime} \tilde{A}\right)$ by a similar computation. From this it follows readily that $e_{\gamma} \tilde{A} \tilde{B}^{\prime} e_{\gamma^{\prime}}=e_{\gamma} \widetilde{B}^{\prime} \tilde{A} e_{\gamma^{\prime}}$ for all $\gamma, \gamma^{\prime} \in \Gamma$, and thus $\tilde{B}^{\prime}$ lies in $\tilde{M}^{\prime}$. We have now proved that the isomorphism $\theta_{\gamma, \gamma}$ is onto $M^{\prime}$. Let $\phi=\theta_{\gamma, \gamma}^{-1}$.

Now let $\tilde{A}$ be arbitrary in $\tilde{M}^{\prime \prime}$. For $B^{\prime}$ arbitrary in $M^{\prime}$ let $\widetilde{B}^{\prime}=\phi^{-1}\left(B^{\prime}\right)$ and consider $\theta_{\gamma, \gamma^{\prime}}(\tilde{A}) B^{\prime}=\theta_{\gamma, \gamma^{\prime}}(\tilde{A}) \theta_{\gamma^{\prime}, \gamma^{\prime}}\left(\tilde{B}^{\prime}\right)=V_{\gamma}^{-1} e_{\gamma} \tilde{A} e_{\gamma^{\prime}} V_{\gamma^{\prime}} V_{\gamma^{\prime}}^{-1} e_{\gamma^{\prime}} \widetilde{B}^{\prime} e_{\gamma^{\prime}} V_{\gamma^{\prime}}$ $=V_{\gamma}^{-1} e_{\gamma} \tilde{A} \tilde{B}^{\prime} e_{\gamma^{\prime}} V_{\gamma^{\prime}}=\theta_{\gamma, \gamma^{\prime}}\left(\tilde{A} \widetilde{B}^{\prime}\right)=\theta_{\gamma, \gamma^{\prime}}\left(\widetilde{B}^{\prime} \tilde{A}\right)=B^{\prime} \theta_{\gamma, \gamma^{\prime}}(\tilde{A})$ showing that $\theta_{\gamma, \gamma^{\prime}}(\tilde{A})$ lies in $M^{\prime \prime}=M$ for all $\gamma, \gamma^{\prime} \in \Gamma$, and hence $\tilde{A}$ lies in $\tilde{M}$. Therefore, we have proved that $\tilde{M}=\tilde{M}^{\prime \prime}$ is a ring of operators on the Hilbert space $\tilde{H}$ such that $\tilde{M}^{\prime}$ is isomorphic to $M^{\prime}$. Also, it is clear that for each $\gamma \in \Gamma, V_{\gamma}$ is a linear isometry mapping $\tilde{M}_{e_{\gamma} \widetilde{H}}$ onto $M$.

To complete the proof of the theorem it is necessary only to show the con- 
tinuity properties of $\phi$ and $\phi^{-1}$. From the computations of the previous page we have $\phi\left(A^{\prime}\right)=\sum_{\gamma \in \Gamma} e_{\gamma} V_{\gamma} A^{\prime} V_{\gamma}^{-1} e_{\gamma}$, and thus for $\tilde{x} \in \tilde{H}, \tilde{y} \in \tilde{H}$,

$$
\begin{aligned}
\left(\phi\left(A^{\prime}\right) \tilde{x}, \tilde{y}\right) & =\sum_{\gamma \in \Gamma}\left(e_{\gamma} V_{\gamma} A^{\prime} V_{\gamma}^{-1} e_{\gamma} \tilde{x}, \tilde{y}\right) \\
& =\sum_{\gamma \in \Gamma}\left(A^{\prime} V_{\gamma}^{-1} e_{\gamma} \tilde{x}, V_{\gamma}^{-1} e_{\gamma} \tilde{y}\right)
\end{aligned}
$$

plus the equation

$$
\left\|\phi\left(A^{\prime}\right) \tilde{x}\right\|^{2}=\sum_{\gamma \in \Gamma}\left\|A^{\prime} V_{\gamma}^{-1} e_{\gamma} \tilde{x}\right\|^{2} .
$$

Now, letting $\left\{\tilde{x}_{n}\right\},\left\{\tilde{y}_{n}\right\}, n$ an integer, be collections of vectors in $\tilde{H}$ such that $\sum_{n=1}^{\infty}\left\|\tilde{x}_{n}\right\|^{2}<\infty, \sum_{n=1}^{\infty}\left\|\tilde{y}_{n}\right\|^{2}<\infty$, we get by the above equations that

and

$$
\sum_{n=1}^{\infty}\left\|\phi\left(A^{\prime}\right) \tilde{x}_{n}\right\|^{2}=\sum_{n=1}^{\infty} \sum_{\gamma \in \Gamma}\left\|A^{\prime} V_{\gamma}^{-1} e_{\gamma} \tilde{x}_{n}\right\|^{2}
$$

$$
\sum_{n=1}^{\infty}\left(\phi\left(A^{\prime}\right) \tilde{x}_{n}, \tilde{y}_{n}\right)=\sum_{n=1}^{\infty} \sum_{\gamma \in \Gamma}\left(A^{\prime} V_{\gamma}^{-1} e_{\gamma} \tilde{x}_{n}, V_{\gamma}^{-1} e_{\gamma} \tilde{y}_{n}\right) ;
$$

which proves that $\phi$ is continuous in both the strongest and the $\sigma$-weak topologies. (Since clearly $\sum_{n=1}^{\infty} \sum_{\gamma \in \Gamma}\left\|V_{\gamma}^{-1} e_{\gamma} \tilde{x}_{n}\right\|^{2}=\sum_{n=1}^{\infty}\left\|\tilde{x}_{n}\right\|^{2}<\infty$, with the same computation for $\left\{\tilde{y}_{n}\right\}$.)

Now let $\left\{x_{n}\right\},\left\{y_{n}\right\}, n$ an integer, be collections of vectors in $H$ such that $\sum_{n=1}^{\infty}\left\|x_{n}\right\|^{2}<\infty, \sum_{n=1}^{\infty}\left\|y_{n}\right\|^{2}<\infty$. By the above equations,

and

$$
\sum_{n=1}^{\infty}\left(\phi\left(A^{\prime}\right) V_{\gamma} x_{n}, V_{\gamma} y_{n}\right)=\sum_{n=1}^{\infty}\left(A^{\prime} x_{n}, y_{n}\right)
$$

$$
\sum_{n=1}^{\infty}\left\|\phi\left(A^{\prime}\right) V_{\gamma} x_{n}\right\|^{2}=\sum_{n=1}^{\infty}\left\|A^{\prime} x_{n}\right\|^{2},
$$

showing that $\phi^{-1}$ is continuous in the strongest and $\sigma$-weak topologies. By specialization of the previous equations, $\left\|\phi\left(A^{\prime}\right) V_{\gamma} x\right\|^{2}=\left\|A^{\prime} x\right\|^{2}$ and $\left(A^{\prime} x, y\right)$ $=\left(\phi\left(A^{\prime}\right) V_{\gamma} x, V_{\gamma} y\right)$, and $\phi^{-1}$ is clearly continuous in the strong and weak topologies. This completes the proof of the theorem.

\section{Chapter II. A Representation theOREM For Linear functionals}

2.1. Introduction. In $[2$, p. 30$]$ is proved a representation theorem for positive linear functionals on countably decomposable, "essentially finite" rings of operators. In this chapter, this theorem is generalized to semifinite rings, and is applied to problems of continuity of the trace. 


\subsection{The representation theorem.}

Definition 2.1. A positive linear functional $\omega$ on a ring $M$ will be called strictly positive if $\omega(E)=0$, for $E$ a projection in $M$, implies $E=0$.

LEMMA 2.1.1. Let $\omega$ be a strictly positive countably additive, linear functional on a ring $M$. If there is a vector $z$ such that $\left[M^{\prime} z\right]=H$ and if $M^{\prime}$ is finite, then there exists a vector $y$ such that $\omega(A)=(A y, y)$ for all $A$ in $M$.

Proof. By Lemma 1.1.2, $M$ is countably decomposable, so that we can proceed as in Lemma 1.2.1 to obtain countable sequences $\left\{E_{n}\right\},\left\{E_{n}^{\prime}\right\}, n$ an integer, of orthogonal projections such that $\omega \sim\left(E_{n}, x_{n}\right)$ for vectors $\left\{x_{n}\right\}$. By hypothesis $M^{\prime}$ is finite and hence $[M z]$ is finite. But Lemma 1.2.4 implies that $\left[M^{\prime} z\right]$ is finite, and with it $M$ is finite.

Continuing as in Lemma 1.2.1, we obtain $I=\sum_{n=1}^{\infty} E_{n}$. Define $F_{n}$ $=\sum_{k=1}^{n} E_{k}$ and assume that the vectors $\left\{y_{k}\right\}$ have been defined for $k \leqq n$, with $x_{1}=y_{1}, F_{k} y_{k+p}=y_{k}$ for $k+p \leqq n, \omega \sim\left(F_{n}, y_{n}\right)$. Also, if $F_{n}^{\prime}=P_{\left[M y_{n}\right]}$, $F_{n}^{\prime} \perp E_{n+p}^{\prime}, p>0$.

We now proceed to the induction step. As in $[2$, p. 34] we get, for arbitrary $A$ in $M$ :

$$
\begin{aligned}
\omega\left(F_{n+1} A^{*} A F_{n+1}\right)= & \omega\left(\left(F_{n}+E_{n+1}\right) A^{*} A\left(F_{n}+E_{n+1}\right)\right) \\
= & \omega\left(F_{n} A^{*} A F_{n}\right)+\omega\left(E_{n+1} A^{*} A F_{n}\right)+\omega\left(F_{n} A^{*} A E_{n+1}\right) \\
& +\omega\left(E_{n+1} A^{*} A E_{n+1}\right) \\
\leqq & \omega\left(F_{n} A^{*} A F_{n}\right)+\omega\left(E_{n+1} A^{*} A E_{n+1}\right)+2\left|\omega\left(F_{n} A^{*} A E_{n+1}\right)\right| \\
\leqq & \omega\left(F_{n} A^{*} A F_{n}\right)+\omega\left(E_{n+1} A^{*} A E_{n+1}\right) \\
& +2\left(\omega\left(F_{n} A^{*} A F_{n}\right) \omega\left(E_{n+1} A^{*} A E_{n+1}\right)\right)^{1 / 2} \\
\leqq & 2\left[\omega\left(F_{n} A^{*} A F_{n}\right)+\omega\left(E_{n+1} A^{*} A E_{n+1}\right)\right] \\
\leqq & 2\left[\left(A y_{n}, A y_{n}\right)+\left(A x_{n+1}, A x_{n+1}\right)\right] \\
\leqq & 2\left(A\left(y_{n}+x_{n+1}\right), A\left(y_{n}+x_{n+1}\right)\right) .
\end{aligned}
$$

(The cross terms like $\left(A x_{n+1}, A y_{n}\right)$ vanish since $\left[M y_{n}\right] \subseteq F_{n}^{\prime} H \perp E_{n=1}^{\prime} H$ $=\left[M x_{n+1}\right]$.) Thus, if $y_{n+1}^{\prime}=2\left(y_{n}+x_{n+1}\right)$, then $\omega \ll\left(F_{n+1}, y_{n+1}^{\prime}\right)$ by Definition 1.2. Clearly $F_{n+1} y_{n+1}^{\prime}=y_{n+1}^{\prime}=\left(F_{n}^{\prime}+E_{n+1}^{\prime}\right) y_{n+1}^{\prime}$, and by Lemma 1.2 .2 , there exists a vector $y^{\prime \prime}$ satisfying $\omega \sim\left(F_{n+1}, y^{\prime \prime}\right)$ and lying in the manifold $\left[F_{n+1} M F_{n+1} y_{n+1}^{\prime}\right] \cap\left[M^{\prime} F_{n+1} y_{n+1}^{\prime}\right]=F_{n+1}\left\{\left[M y_{n+1}^{\prime}\right] \cap\left[M^{\prime} y_{n+1}^{\prime}\right]\right\}=\left[M y_{n+1}^{\prime}\right]$ $\cap\left[M^{\prime} y_{n+1}^{\prime}\right] \subseteq\left\{\left[M x_{n+1}\right]+\left[M y_{n}\right]\right\} \cap\left\{\left[M^{\prime} x_{n+1}\right]+\left[M^{\prime} y_{n}\right]\right\} \subseteq\left(E_{n+1}^{\prime}+F_{n}^{\prime}\right)\left(E_{n+1}\right.$ $\left.+F_{n}\right) H=F_{n+1}\left(F_{n}^{\prime}+E_{n+1}^{\prime}\right) H$. Then, if $A$ is arbitrary in $M, \omega\left(F_{n} A^{*} A F_{n}\right)$ $=\left(A^{*} A y_{n}, y_{n}\right)=\left\|A y_{n}\right\|^{2}=\left(F_{n} A^{*} A F_{n} y^{\prime \prime}, y^{\prime \prime}\right)=\left\|A F_{n} y^{\prime \prime}\right\|^{2}$, showing that the mapping $V^{\prime}$ defined as $V^{\prime} A F_{n} y^{\prime \prime}=A y_{n}$ can be extended to a partial isometry in $M^{\prime}$ taking $\left[M F_{n} y^{\prime \prime}\right]$ into $\left[M y_{n}\right]$. Also, since $\left[M F_{n} y^{\prime \prime}\right] \subseteq\left[M y^{\prime \prime}\right] \subseteq\left(F_{n}^{\prime}+E_{n+1}^{\prime}\right) H$ and $\left[M y_{n}\right]=F_{n}^{\prime} H \subseteq\left(F_{n}^{\prime}+E_{n+1}^{\prime}\right) H, V^{\prime}$ can be extended to the partial isometry $V_{n+1}^{\prime}$ such that $V_{n+1}^{\prime *} V_{n+1}^{\prime}=V_{n+1}^{\prime} V_{n+1}^{\prime *}=E_{n+1}^{\prime}+F_{n}^{\prime}$ ( $M^{\prime}$ is finite). 
Now let $y_{n+1}=V_{n+1}^{\prime} y^{\prime \prime}, F_{n+1}^{\prime}=P_{\left[M y_{n+1}\right]}$. Then, $\left(A y_{n+1}, y_{n+1}\right)=\left(A V_{n+1}^{\prime} y^{\prime \prime}\right.$, $\left.V_{n+1}^{\prime} y^{\prime \prime}\right)=\left(A V_{n+1}^{\prime *} V_{n+1}^{\prime} y^{\prime \prime}, \quad y^{\prime \prime}\right)=\left(A\left(F_{n}^{\prime}+E_{n+1}^{\prime}\right) y^{\prime \prime}, \quad y^{\prime \prime}\right)=\left(\begin{array}{ll}A y^{\prime \prime}, & y^{\prime \prime}\end{array}\right)$ $=\omega\left(F_{n+1} A F_{n+1}\right)$, or

$$
\omega \sim\left(F_{n+1}, y_{n+1}\right) .
$$

Also, $F_{n+1}^{\prime} H=\left[M y_{n+1}\right]=\left[M V_{n+1}^{\prime} y^{\prime \prime}\right]=V_{n+1}^{\prime}\left[M y^{\prime \prime}\right] \subseteq\left(F_{n}^{\prime}+E_{n+1}^{\prime}\right) H$, and $F_{n} y_{n+1}$ $=F_{n} V_{n+1}^{\prime} y^{\prime \prime}=V_{n+1}^{\prime} F_{n} y^{\prime \prime}=y_{n}$, proving that $F_{n+1}^{\prime} \perp E_{n+1+p}, p>0$, and completing the induction step.

We now have, by the above induction argument, a sequence of vectors $\left\{y_{n}\right\}$ and a sequence of projections $\left\{F_{n}\right\}$ such that $\omega \sim\left(F_{n}, y_{n}\right)$ and $F_{n}$ $=\sum_{k=1}^{n} E_{k}$. But the computation

$$
\begin{aligned}
\left\|y_{n+p}-y_{n}\right\|^{2} & =\left\|F_{n+p} y_{n+p}-F_{n} y_{n+p}\right\|^{2}=\left\|\left(F_{n+p}-F_{n}\right) y_{n+p}\right\|^{2} \\
& =\left(\left(F_{n+p}-F_{n}\right) y_{n+p}, y_{n+p}\right)=\omega\left(F_{n+p}-F_{n}\right) \\
& =\omega\left(\sum_{k=n+1}^{n+p} E_{k}\right)=\sum_{k=n+1}^{n+p} \omega\left(E_{k}\right)
\end{aligned}
$$

shows that the sequence $\left\{y_{n}\right\}$ is cauchy, and thus there exists a vector $y$ such that $\left\{y_{n}\right\}$ converges to it. Also, we have, for $A$ in $M$ :

$$
\begin{aligned}
\left|\omega(A)-\omega\left(F_{n} A F_{n}\right)\right| & \leqq\left|\omega(A)-\omega\left(A F_{n}\right)\right|+\left|\omega\left(A F_{n}\right)-\omega\left(F_{n} A F_{n}\right)\right| \\
& \leqq\left(\omega\left(A A^{*}\right) \omega\left(I-F_{n}\right)\right)^{1 / 2}+\left(\omega\left(F_{n} A^{*} A F_{n}\right) \cdot \omega\left(I-F_{n}\right)\right)^{1 / 2} \\
& \leqq\left[\left(\omega\left(A A^{*}\right)\right)^{1 / 2}+\left(\omega\left(F_{n} A^{*} A F_{n}\right)\right)^{1 / 2}\right] \cdot\left(\omega\left(I-F_{n}\right)\right)^{1 / 2} \\
& \leqq\left[\left(\omega\left(A A^{*}\right)\right)^{1 / 2}+\left\|A y_{n}\right\|\right] \cdot\left(\omega\left(\sum_{k=n+1}^{\infty}\left(E_{k}\right)\right)\right)^{1 / 2} \\
& \leqq\left[\left(\omega\left(A A^{*}\right)\right)^{1 / 2}+\left\|A y_{n}\right\|\right] \cdot\left(\sum_{k=n+1}^{\infty} \omega\left(E_{k}\right)\right)^{1 / 2}
\end{aligned}
$$

which implies $(A y, y)=\lim _{n=\infty}\left(A y_{n}, y_{n}\right)=\lim _{n=\infty} \omega\left(F_{n} A F_{n}\right)=\omega(A)$.

Lемма 2.1.2. If $\omega$ is a countably additive, positive linear functional on a countably decomposable ring $M$, then there exists a projection $E$ in $M$ such that $\omega\left(E^{\perp}\right)=0$ and such that $\omega$ is strictly positive on the ring $M_{E H}$.

Proof. Choose, by Zorn's Lemma, a maximal collection $\left\{F_{n}\right\}, n \in \Gamma$, of orthogonal projections in $M$ such that $\omega\left(F_{n}\right)$ are all zero. Because of countable decomposability of $M$ we may assume that $\Gamma$ is the set of positive integers.

Let $F=\sum_{n=1}^{\infty} F_{n}$. Using countable additivity of $\omega, \omega(F)=\sum_{n=1}^{\infty} \omega\left(F_{n}\right)=0$. We now can show that $(I-F)$ is the $E$ of the lemma.

Assume that $G$ is a projection in $M_{(I-F) H}$ such that $\omega(G)=0$. But then $G$ could be added to our maximal collection $\left\{F_{n}\right\}$, thus contradicting our argument and proving $\omega$ is strictly positive. 
THEOREM 5. Let $\omega$ be a countably additive, positive linear functional on a ring $M$. If there exists a vector $z$ such that $[M z]$ is finite and if, for $A$ in $M$, $A z=0$ implies $\omega(A)=0$, then there exists a vector $x$ in the manifold $[M z] \cap\left[M^{\prime} z\right]$ such that $\omega \sim(I, x)$.

Proof. Let $G=P_{\left[M^{\prime} z\right]}$ and consider the linear functional $(A z, z)$ on the ring $M_{G H}$. If $(F z, z)=0$ for $F$ a projection in $M_{G H}$, then clearly $F_{z}=0$ and consequently $F G=F=0$, showing that $M_{G H}$ is countably decomposable by Lemma 1.1.2. Now, invoking Lemma 2.1.2, there is a projection $E$ in $M_{G H}$ such that $\omega(G-E)=0$ and such that $\omega$ is strictly positive on $M_{E H}$. Also, notice that $G^{\perp} z=0$ implies that $\omega\left(G^{\perp}\right)=0$ by hypothesis. Thus we have a projection $E$ in $M$ such that $\omega$ is strictly positive on $M_{E H}$ and such that $\omega\left(E^{\perp}\right)=\omega(G-E)$ $+\omega\left(G^{\perp}\right)=0$.

Let $E^{\prime}=P_{[M E z]}$ (finite, by hypothesis) and consider the ring $M_{E E^{\prime} H}$. The mapping $A \rightarrow A E^{\prime}$ is an isomorphism of $M_{E H}$ onto $M_{E E^{\prime} H}$, since if $A E^{\prime}=0$, then $A E^{\prime} E z=A E z=0$ which in turn implies $A=0$ by $A \in M_{E H} \subseteq M_{G H}$. Therefore we can introduce the linear functional $\phi$ on $M_{E E^{\prime} H}$ by $\phi\left(A E^{\prime}\right)=\omega(A)$. $\phi$ is clearly a strictly positive, countably additive, linear functional on $M_{E E^{\prime} H}$, with $M_{E E^{\prime} H}^{\prime}$ finite, and $\left[M_{E E^{\prime} H}^{\prime} E z\right]=\left[E^{\prime} M^{\prime} E^{\prime} E z\right]=E E^{\prime}\left[M^{\prime} z\right]=E E^{\prime} G H$ $=E E^{\prime} H$.

Now, using Lemma 2.1.1, we get a vector $y$ lying in the manifold $E E^{\prime} H \subseteq E^{\prime} G H=[M E z] \cap\left[M^{\prime} z\right] \subseteq[M z] \cap\left[M^{\prime} z\right]$, such that $\phi\left(A E^{\prime}\right)=\omega(A)$ $=\left(A E^{\prime} y, y\right)=(A y, y)$ for $A$ in $M_{E H}$. But then the inequality $\left|\omega\left(A E^{\perp}\right)\right|^{2}$ $\leqq \omega\left(A A^{*}\right) \omega\left(E^{\perp}\right)=0$ (plus a similar one for $\left(E^{\perp} A\right)$ ) proves that for $A$ in $M$,

$$
\begin{aligned}
\omega(A) & =\omega(E A E)+\omega(E A E \perp)+\omega(E \perp A E)+\omega(E \perp A E \perp)=\omega(E A E) \\
& =(E A E y, y) \\
& =(A y, y),
\end{aligned}
$$

thus completing the proof of the theorem.

\subsection{Applications of Theorem 5 .}

Theorem 6. Let $\left(M, M^{\prime}\right)$ and $\left(\tilde{M}, \tilde{M}^{\prime}\right)$ be finite rings on the spaces $H$ and $\tilde{H}$, with joint cyclic vectors $z$ and $\tilde{z}$. If $\phi$ is $a^{*}$-isomorphism of $M$ onto $\tilde{M}$, then there exists a linear isometry $W$ mapping $H$ onto $\tilde{H}$ with $\phi(A)=W A W^{-1}$, for $A$ in $M$.

Proof. Consider the linear functional $\omega$ defined on $M$ by $\omega(A)=(\phi(A) \tilde{z}, \tilde{z})$ for $A$ in $M$. If $A z=0$, then $A=0$ by $\left[M^{\prime} z\right]=H$, showing that $\omega$ satisfies all the hypotheses of Theorem 5. Let $y$ be the vector in $H$ such that $\omega(A)$ $=(A y, y)$. Then $\|A y\|^{2}=\omega\left(A^{*} A\right)=\|\phi(A) \tilde{z}\|^{2}$, and $A y=0$ implies $\phi(A) \tilde{z}=0$, with finally $A=0$. Thus $\left[M^{\prime} y\right]=H=\left[M^{\prime} z\right]$ and $[M y] \sim[M z]=H$ by Lemma 9.3.3, p. 179 of $[5]$.

Let $V^{\prime}$ be the partial isometry in $M^{\prime}$ defined by $V^{\prime}[M y]=\left[M V^{\prime} y\right]=H$ and define $x=V^{\prime} y$. Now $[M x]=H$ and $\|A x\|^{2}=\left\|A V^{\prime} y\right\|^{2}=\left(A * A V^{*} V^{\prime} y, y\right)$ $=\left(A^{*} A y, y\right)=\|A y\|^{2}=\|\phi(A) \bar{z}\|^{2}$ proves that we can define a linear isometry 
$W$ from $H$ onto $\tilde{H}$ such that $W A x=\phi(A) \tilde{z}$. Furthermore, if $\widetilde{B}$ arbitrary in $\tilde{M}$, then $W A W^{-1} \tilde{B} \tilde{z}=W A \phi^{-1}(\tilde{B}) x=\phi(A) \tilde{B} \tilde{z}$ proves that $\phi(A)=W A W^{-1}$ for $A$ in $M$.

THEOREM 7. Let $\omega$ be a countably additive, positive, linear functional on a finite ring $M$. If the coupling operator $C$ of the pair $\left(M, M^{\prime}\right)$ is bounded $\left(C_{1}\right.$ is bounded), then there exists a finite set $z_{1}, z_{2}, \cdots, z_{n}$ of vectors such that $\omega(A)$ $=\sum_{k=1}^{n}\left(A z_{k}, z_{k}\right)$ for $A$ in $M$. In fact, if the $C_{1}$ of Definition 1.6 is bounded above by $m P_{1,1}, m$ an integer, then $n \leqq m$.

Proof. We first consider the case where $C_{1} \leqq P_{1,1}$. As in the proof of Theorem 1, we use Lemma 1.2.7 and Lemma 1.2.8 to get a collection $\left\{P_{\alpha}^{\prime}\right\}, \alpha \in \Gamma$, of orthogonal projections in the center of $M$ such that either $\left[M^{\prime} x_{\alpha}\right]=P_{\alpha}^{\prime} H$ or $\left[M x_{\alpha}\right]=P_{\alpha}^{\prime} H$ with $\sum_{\alpha \in \Gamma} P_{\alpha}^{\prime}=I$. But by the definition of $C_{1}$, if $\left[M x_{\alpha}\right]$ $=P_{\alpha}^{\prime} H$ then $P_{\alpha}^{\prime} \leqq P_{1,1}$ by Lemma 1.2.4, and $\left[M^{\prime} x_{\alpha}\right]^{\natural}=C_{1}^{-1}\left[M x_{\alpha}\right]^{\natural}=C_{1}^{-1} P_{\alpha}^{\prime}$ $\geqq P_{\alpha}^{\prime}$, proving that $P_{\alpha}^{\prime}=\left[M^{\prime} x_{\alpha}\right]$ and yielding us a collection of operators $\left\{P_{\alpha}^{\prime}\right\}$ in the center of $M$ such that $\sum_{\alpha \in \Gamma} P_{\alpha}^{\prime}=I$ and such that each is generated by a vector $x_{\alpha}$ and $M^{\prime}$.

Let $\Gamma^{\prime}$ be the set of $\alpha \in \Gamma$ such that $\omega\left(P_{\alpha}^{\prime}\right) \neq 0$. It follows from linearity of $\omega$ that $\Gamma^{\prime}$ is countable and thus there is obviously no loss in generality in assuming $\sum_{\alpha \in \Gamma^{\prime}}\left\|x_{\alpha}\right\|^{2}<\infty$. In fact, by the orthogonality of the $\left\{P_{\alpha}^{\prime}\right\}$, $\alpha \in \Gamma$, there exists a vector $x=\sum_{\alpha \in \Gamma^{\prime}} x_{\alpha}$. If $P^{\prime}=\sum_{\alpha \in \Gamma} P_{\alpha}^{\prime}$, then, for $A$ in $M, \omega(A)=\omega\left(P^{\prime} A\right)+\omega\left(A P^{\prime \perp}\right)=\omega\left(A P^{\prime}\right)$, by $\left|\omega\left(A P^{\prime \perp}\right)\right|^{2} \leqq \omega\left(A A^{*}\right) \omega\left(P^{\prime \perp}\right)=0$. Also, $A x=0$ implies $0=A P_{\alpha}^{\prime} x=A P_{\alpha}^{\prime} x\left(\alpha \in \Gamma^{\prime}\right)$ which in turn implies $A P_{\alpha}^{\prime}=0$ for $\alpha \in \Gamma^{\prime}$. But $A P_{\alpha}^{\prime}=0, \alpha \in \Gamma^{\prime}$, yields $A P^{\prime}=0$ and finally $\omega(A)=\omega\left(A P^{\prime}\right)=0$. Thus we have proved that $\omega$ and $x$ satisfy the requirements of Theorem 5 , once we notice that $[M x]$ is finite by Lemma 1.2.4. This completes the proof of the case $C_{1} \leqq P_{1,1}$.

We now consider the case $C_{1} \leqq m P_{1,1}$ for $m$ an integer. Again we pick orthogonal projections $\left\{P_{\alpha}^{\prime}\right\}, \alpha \in \Gamma^{\prime}$, in the center of $M$ such that $\sum_{\alpha \in \Gamma^{\prime}} P_{\alpha}^{\prime}$ $=I$ with either $P_{\alpha}^{\prime} H=\left[M^{\prime} x_{\alpha}\right]$ or $P_{\alpha}^{\prime} H=\left[M x_{\alpha}\right]$. Now, with $\Gamma$ equal to the positive integers less than $m+1$, apply Theorem 4 to $M^{\prime}$, obtaining rings $\tilde{M}^{\prime}$ and $\tilde{M}$ with equivalent projections $e_{k}, k \in \Gamma$, in $\tilde{M}^{\prime}$ such that $\sum_{k=1}^{m} e_{k}=I$, $e_{k} M^{\prime} e_{k}=V_{k} \tilde{M}^{\prime} V_{k} e_{k}$ via the isometries $V_{k}$. We also get an isomorphism $\phi$ of $M$ onto $\tilde{M}$ such that

$$
\phi(A)=\sum_{k=1}^{m} V_{k} A V_{k}^{-1} e_{k}
$$

for $A$ in $M$.

Let $F_{\alpha}=P_{\left[M^{\prime} x_{\alpha}\right]}, \tilde{F}_{\alpha}=P_{\left[\tilde{M}^{\prime} V_{1} x_{\alpha}\right]}, \tilde{F}_{\alpha}^{\prime}=P_{\left[\widetilde{M} V_{1} x_{\alpha}\right]}$. From the proof of Theorem $4, \theta_{1,1}\left(\tilde{F}_{\alpha}\right)=\phi^{-1}\left(\widetilde{F}_{\alpha}\right)=V_{1}^{-1} e_{1} \widetilde{F}_{\alpha} e_{1} V_{1}$, and thus $\phi^{-1}\left(\tilde{F}_{\alpha}\right) H=V_{1}^{-1} e_{1} \tilde{F}_{\alpha} e_{1} H_{1}$ $=V_{1}^{-1} e_{1} \widetilde{F}_{\alpha} \tilde{H}=V_{1}^{-1} e_{1}\left[\tilde{M}^{\prime} V_{1} x_{\alpha}\right]=V_{1}^{-1}\left[e_{1} \tilde{M}^{\prime} e_{1} V_{1} x_{\alpha}\right]=V_{1}^{-1}\left[V_{1} M^{\prime} V_{1}^{-1} V_{1} x_{\alpha}\right]=\left[M^{\prime} x_{\alpha}\right]$ $=F_{\alpha} H$, proving that

$$
\phi\left(F_{\alpha}\right)=\tilde{F}_{\alpha} .
$$


We now consider the $x_{\alpha}$ such that $\left[M x_{\alpha}\right]=P_{\alpha}^{\prime} H . \phi\left(P_{\alpha}^{\prime}\right) e_{1} \tilde{H}=V_{1} P_{\alpha}^{\prime} V_{1}^{-1} e_{1} \tilde{H}$ $=V_{1} P_{\alpha}^{\prime} H=V_{1}\left[M x_{\alpha}\right] \quad$ and $\tilde{F}_{\alpha}^{\prime} \tilde{H}=\left[\tilde{M} V_{1} x_{\alpha}\right]=\left[V_{1} M V_{1}^{-1} e_{1} V_{1} x_{\alpha}\right]=V_{1}\left[M x_{\alpha}\right]$, showing that

$$
\phi\left(P_{\alpha}^{\prime}\right) e_{1}=\tilde{F}_{\alpha}^{\prime}
$$

But by definition of the coupling operator, $P_{\alpha}^{\prime}=\left[M x_{\alpha}\right]^{\natural}=C_{1}\left[M^{\prime} x_{\alpha}\right]^{\natural}$ $=C_{1}\left(F_{\alpha}\right)^{\natural}$; and by the continuity properties of $\phi, \phi\left(P_{\alpha}^{\prime}\right)=\phi\left(C_{1}\right) \cdot \phi\left(\left(F_{\alpha}\right)^{\natural}\right)$ $=\phi\left(C_{1}\right) \cdot\left(\left(\tilde{F}_{\alpha}\right)^{\natural}\right)$. Also, if $\tilde{C}$ is the coupling operator for $\tilde{M}, \tilde{M}^{\prime}$, then $\left(\tilde{F}_{\alpha}^{\prime}\right)^{\natural}$ $=\left[\tilde{M} V_{1} x_{\alpha}\right]^{\natural}=\tilde{C}_{1}\left[\tilde{M}^{\prime} V_{1} x_{\alpha}\right]^{\natural}=\tilde{C}_{1}\left(\tilde{F}_{\alpha}\right)^{\natural}$, and we get, by combining these two equations (using $\left.\left(\tilde{F}_{\alpha}^{\prime}\right)^{\natural}=\phi\left(P_{\alpha}^{\prime}\right)\left(e_{1}\right)^{\natural}=(1 / m) \phi\left(P_{\alpha}^{\prime}\right)\right), \quad(1 / m) \phi\left(P_{\alpha}^{\prime}\right)=\tilde{C}_{1}\left(\tilde{F}_{\alpha}\right)^{\natural}$ $=(1 / m) \phi\left(C_{1}\right) \cdot\left(\tilde{F}_{\alpha}\right)^{\natural}$. But clearly $\left(\tilde{F}_{\alpha}\right)^{\natural}$ has an inverse with respect to $\phi\left(P_{\alpha}^{\prime}\right)$ and we get finally that

$$
\widetilde{C}_{1} \phi\left(P_{\alpha}^{\prime}\right)=\phi\left(C_{1}\right) \phi\left(P_{\alpha}^{\prime}\right) \cdot \frac{1}{m} \leqq \phi\left(P_{\alpha}^{\prime}\right) .
$$

For those $\alpha \in \Gamma$ such that $\left[M^{\prime} x_{\alpha}\right]=F_{\alpha} H=P_{\alpha}^{\prime} H$, we get $\phi\left(F_{\alpha}\right)=\tilde{F}_{\alpha}$ $=\phi\left(P_{\alpha}^{\prime}\right)$ or $\phi\left(P_{\alpha}^{\prime}\right) \widetilde{H}=\widetilde{F}_{\alpha} \tilde{H}=\left[\tilde{M}^{\prime} V_{1} x_{\alpha}\right]$. It is now clear from Lemma 1.2.5 that here too $\widetilde{C}_{1} \phi\left(P_{\alpha}^{\prime}\right) \leqq \phi\left(P_{\alpha}^{\prime}\right)$. Combining this result with the preceding paragraph, we obtain finally the inequality

$$
\widetilde{C}_{1} \leqq \widetilde{P}_{1,1},
$$

as $\sum_{\alpha \in \Gamma} \phi\left(P_{\alpha}^{\prime}\right)=I$.

We now introduce the linear functional $\tilde{\omega}$ on $\tilde{M}$ by defining $\tilde{\omega}(\tilde{A})$ $=\omega\left(\phi^{-1}(\tilde{A})\right)$. This functional clearly satisfies all the requirements of the first paragraph of the proof of this theorem, and hence there exists a vector $\tilde{x}$ in $\widetilde{H}$ such that $\tilde{\omega}(\tilde{A})=(\tilde{A} \bar{x}, \tilde{x})$. Then, for $A$ in $M$,

$$
\begin{aligned}
\omega(A) & =\tilde{\omega}(\phi(A))=(\phi(A) \tilde{x}, \tilde{x}) \\
& =\left(\sum_{k=1}^{m} V_{k} A V_{k}^{-1} e_{k} \tilde{x}, \tilde{x}\right) \\
& =\sum_{k=1}^{m}\left(A V_{k}^{-1} e_{k} \tilde{x}, V_{k}^{-1} e_{k} \tilde{x}\right),
\end{aligned}
$$

and the nonzero members of the set $\left\{V_{k}^{-1} e_{k} \tilde{x}\right\}$ form our set $z_{1}, z_{2}, \cdots, z_{n}$, with $n \leqq m$.

Theorem 8. Let $M$ be a finite ring. The mapping $A \rightarrow A^{\natural}$ is continuous in the strong (weak) topology if and only if the coupling operator $C$ is bounded.

Proof. First, assume $C$ is bounded. For any vector $x$, the linear functional $\left(A^{\natural} x, x\right)$ on $M$ clearly satisfies all the requirements of Theorem 7 , and therefore there exists a finite collection of vectors $\left\{z_{\alpha}\right\}$ such that $\left(A^{\natural} x, x\right)$ $=\sum_{k=1}^{n}\left(A z_{k}, z_{k}\right)$. This proves that the trace is weakly continuous. Then, by 
Lemma 8.1, p. 254 of [1], $\left(A^{\natural}\right)^{*}\left(A^{\natural}\right) \leqq\left(A^{*} A\right)^{\natural}$ for all $A$ in $M$, and we get the inequality $\left\|A^{\natural} x\right\|^{2}=\left(\left(A^{\natural}\right)^{*}\left(A^{\natural}\right) x, \quad x\right) \leqq\left(\left(A^{*} A\right)^{\natural} x, \quad x\right)=\sum_{k=1}^{n} \quad\left(A^{*} A z_{k}, \quad z_{k}\right)$ $\leqq \sum_{k=1}^{n}\left\|A z_{k}\right\|^{2}$, which proves that the trace is strongly continuous.

Now, assume $C$ is unbounded. As this means that $C_{1}$ is unbounded, there is clearly no loss of generality in assuming that $M$ and $M^{\prime}$ are both finite. Then, by the spectral theorem, there exists a sequence $\left\{P_{k}\right\}, k$ an integer, of projections in the center of $M$ such that $I=P_{0}>P_{1}>P_{2}>\ldots$, with $C P_{n} \geqq n P_{n}$. Also, we can choose vectors $z_{n} \in\left(P_{n}-P_{n-1}\right) H$ such that $\left\|z_{n}\right\|^{2}=1 / n^{2}$, and since $\sum_{n=1}^{\infty}\left\|z_{n}\right\|^{2}=\sum_{n=1}^{\infty}\left(1 / n^{2}\right)<\infty$, there exists a vector $z=\sum_{n=1}^{\infty} z_{n}$.

For $x$ arbitrary in $H$, define $E(x)=P_{\left[M^{\prime} x\right]}$. Clearly $E(x) P_{n}=E\left(P_{n} x\right)$ for all $n$, and by the definition of $C, E\left(P_{n} x\right)^{\natural}=C^{-1}\left[M P_{n} x\right]^{\natural} \leqq C^{-1} P_{n} \leqq(1 / n) P_{n}$. Also, let $\Omega$ be the collection of all finite sets of vectors $\left\{\left(x_{1}, x_{2}, \cdots, x_{n}\right)\right\}$, $n$ some integer, which is made into a directed set by defining (for $\tau_{1}, \tau_{2} \in \Omega$ ) $\tau_{1} \leqq \tau_{2}$ if $\tau_{1} \subseteq \tau_{2}$.

If $\tau(\in \Omega)$ consists of the above vectors $\left\{x_{k}\right\}$, define $E_{\tau}=\sup _{x_{k} \in \tau} E\left(P_{n+1} x_{k}\right)$ and $F_{\tau}=P_{n+1}-E_{\tau}$. Now, by Theorem $5.4[4$, p. 244] for arbitrary projections $E, \quad F$ in $M, \quad E \cup F-F \sim E-E \cap F$ or $(E \cup F)^{\natural}=E^{\natural}+F^{\natural}-(E \cap F)^{\natural}$ $\leqq E^{\natural}+F^{\natural}$. Then, by a trivial induction argument, we obtain $\left(E_{\tau}\right)^{\natural}$ $\leqq \sum_{k=1}^{n}\left(E\left(P_{n+1} x_{k}\right)^{\natural}\right) \leqq(n /(n+1)) P_{n+1}$ and thus $\left(F_{\tau}\right)^{\natural} \geqq P_{n+1}-(n /(n+1)) P_{n+1}$ $\geqq(1 /(n+1)) P_{n+1}$. Clearly $F_{\tau} x_{k}=0$ and for $\tau^{\prime} \geqq \tau, F_{\tau^{\prime}} x_{k}=0$.

Now, with the above notation, we get a directed set of operators $\left\{A_{\tau}\right\}$, $\tau \in \Omega$, by letting $A_{\tau}=(n+1)^{3} F_{\tau}$, with the following property: If $x$ is arbitrary in $H$, let $\tau_{0}=(x)$ in $\Omega$, and consider $A_{\tau_{0}} x=(n+1)^{3} F_{\tau_{0}} x=0$. As in the above paragraph, $A_{\tau} x=0$ for $\tau \geqq \tau_{0}$, proving that $A_{\tau}$ converges to 0 in the strong topology. But $A_{\tau}^{\natural}=(n+1)^{3} F_{\tau}^{\natural} \geqq(n+1)^{3} \cdot(1 /(n+1)) P_{n+1} \geqq(n+1)^{2}\left(P_{n+1}-P_{n}\right)$ shows that, using the vector $z$ defined above, $\left(A_{\tau}^{\natural} z, z\right) \geqq(n+1)^{2} \cdot\left\|\left(P_{n+1}-P_{n}\right) z\right\|^{2}$ $\geqq(n+1)^{2}\left\|z_{n+1}\right\|^{2}=1$; and thus the directed set $\left\{A_{\tau}^{\natural}\right\}$ does not converge to zero in the weak topology.

Thus we have succeeded in constructing a directed set of operators $\left\{A_{\tau}\right\}$ in $M$ which converge to zero in the strong (and thus in the weak) topology, but with the set $\left\{A_{\tau}^{\natural}\right\}$ not converging to zero in the weak (and thus not in the strong) topology. This success proves that the mapping $A \rightarrow A^{\natural}$ is continuous in neither the strong nor the weak topology, and the proof of this theorem is complete.

\section{Chapter III. Continuity properties of *-ISOMorphisms of Rings}

3.1. Introduction. The main result of this chapter is a condition which determines when a ${ }^{*}$-isomorphism between semifinite rings is given by a spatial isometry, thus generalizing a result of von Neumann in $\$ 3.3$ of [7]. Then, growing out of this result, we obtain conditions for various of the standard topologies to be purely algebraic. Our final result is a theorem on the relationship of a semifinite ring to its commutant.

\subsection{Spatial isomorphism of semifinite rings.}


Definition 3.1. Let $\left(M, M^{\prime}\right),\left(\tilde{M}, \tilde{M}^{\prime}\right)$ be finite rings with coupling operators $C, \tilde{C}$ respectively, and let $\phi$ be a ${ }^{*}$-isomorphism of $M$ onto $\tilde{M}$. Further, as in the proof of Theorem 1, let the coupling operators have the form: $C=\sum_{\alpha \in \Gamma}\left(C_{\alpha}+D_{\alpha}^{-1}\right), \tilde{C}=\sum_{\alpha \in \Gamma}\left(\widetilde{C}_{\alpha}+\widetilde{D}_{\alpha}^{-1}\right)$. If there exist decompositions of $C$ and $\widetilde{C}$ such that $\phi\left(C_{\alpha}\right)=\widetilde{C}_{\alpha}$ and $\phi\left(D_{\alpha}\right)=\widetilde{D}_{\alpha}$, then we write $\phi(C)=\widetilde{C}$.

Lemma 3.2.1. Let $\left(M, M^{\prime}\right),\left(\tilde{M}, \tilde{M}^{\prime}\right)$ be finite rings on Hilbert spaces $H, \tilde{H}$ with coupling operators $C, \widetilde{C}$ respectively. Further, let $z, \tilde{z}$ be vectors in $H, \widetilde{H}$ respectively such that $[\mathrm{Mz}]=H,[\widetilde{M} \tilde{z}]=\widetilde{H}$. If $\phi$ is a ${ }^{*}$-isomorphism of $M$ onto $\tilde{M}$ such that $\phi(C)=\tilde{C}$, then there exists an isometry $W$ of $H$ onto $\widetilde{H}$ such that $W A W^{-1}=\phi(A)$ for $A$ in $M$.

Proof. If $G=P_{\left[M^{\prime} z\right]}$, then $G^{\natural} C=C\left[M^{\prime} z\right]^{\natural}=[M z]^{\natural}=I$, and thus $I=\phi(I)$ $=\phi\left(G^{\natural}\right) \cdot \phi(C)=\phi(G)^{\natural} \tilde{C}$. But, since $[\tilde{M} \bar{z}]^{\natural}=\widetilde{C}\left[\tilde{M}^{\prime} \tilde{z}\right]^{\natural}=\tilde{I}$, we get $\phi(G)^{\natural}=[\tilde{M} \tilde{z}]^{\natural}$. Thus we can select a vector $\tilde{x}$ in $\widetilde{H}$ such that $\left[\tilde{M}^{\prime} \tilde{x}\right]=\phi(G) \widetilde{H}$ and $[\tilde{M} \tilde{x}]=\widetilde{H}$. Also, $\left[M_{\left[M^{\prime} z\right]} z\right]=\left[M_{\left[M^{\prime} z\right.}^{\prime} z\right]=G H$ and $\left[\tilde{M}_{\left[\widetilde{M}^{\prime} x\right]} \tilde{x}\right]=\left[\tilde{M}_{\left[\tilde{M}^{\prime} \tilde{x}\right)}^{\prime} \tilde{x}\right]=\phi(G) \tilde{H}$, showing that, by Theorem 6 , there exists a linear isometry $W_{0}$ mapping $G H$ onto $\phi(G) \widetilde{H}$ with $\phi(A)=W_{0} A W_{0}^{-1}$ for $A$ in $M_{\left[M^{\prime} z\right]}$.

Now, by Lemmas 4.11, p. $231,4.9$, p. 230 , and 6.4 , p. 235 of [1], we can select a nonzero projection $E$ in $M$ such that $E \leqq G$ and such that $E^{\natural}=(1 / n) P$ for some projection $P$ in the center of $M$. Further, one can choose orthogonal projections $\left\{E_{k}\right\}, 1 \leqq k \leqq n$, such that each $E_{k}$ is equivalent to $E$ and with $\sum_{k=1}^{n} E_{k}=P$. Let $V_{k}, 1 \leqq k \leqq n$, be partial isometries in $M$ such that $V_{k}^{*} V_{k}=E_{k}$ and $V_{k} V_{k}^{*}=E$.

Clearly, the mappings $\phi\left(V_{k}\right)^{*} W_{0} V_{k}$ are isometric from $E_{k} H$ to $\phi\left(E_{k}\right) \tilde{H}$, since $\phi\left(V_{k}^{*}\right) \phi\left(V_{k}\right)=\phi\left(E_{k}\right)$ and $\phi\left(V_{k}\right) \phi\left(V_{k}^{*}\right)=\phi(E)$. Thus we can define the mapping $W=\sum_{k=1}^{n} \phi\left(V_{k}\right)^{*} W_{0} V_{k}$ as an isometric map of $P H$ onto $\phi(P) \tilde{H}$. For $A$ in $M P$ consider

$$
\begin{aligned}
W A W^{-1} & =\sum_{k, s=1}^{n} \phi\left(V_{k}\right)^{*} W_{0} V_{k} A V_{s}^{*} W_{0}^{-1} \phi\left(V_{s}\right) \\
& \left.=\sum_{s, k=1}^{n} \phi\left(V_{k}^{*}\right) \phi\left(V_{k} A V_{s}^{*}\right) \phi\left(V_{s}\right) \quad \text { (since } V_{k} A V_{s}^{*} \text { in } M_{E H}\right) \\
& =\sum_{s, k=1}^{n} \phi\left(V_{k}^{*} V_{k} A V_{s}^{*} V_{s}\right)=\phi\left(\sum_{k, 8=1}^{n}\left(E_{k} A E_{s}\right)\right) \\
& =\phi(A P)=\phi(A) .
\end{aligned}
$$

Thus we have constructed an isometry implementing $\phi$ on $M P$, for a projection $P$ in the center of $M$. From this result, an obvious transfinite induction argument yields a collection $\left\{P_{\alpha}\right\}, \alpha \in \Gamma$, of orthogonal projections in the center of $M$, such that $\sum_{\alpha \in \Gamma} P_{\alpha}=I$ and such that there exist linear isometries $W_{\alpha}$ mapping $P_{\alpha} H$ onto $\phi\left(P_{\alpha}\right) \tilde{H}$ and implementing $\phi$ on $M P_{\alpha}$. Let $W=\sum_{\alpha \in \boldsymbol{\Gamma}} W_{\boldsymbol{\alpha}}$. 
For $\Gamma^{\prime}$ any finite subset of $\Gamma$, we notice that

$$
\begin{aligned}
W\left(A \sum_{\alpha \in \Gamma^{\prime}} P_{\alpha}\right) W^{-1} & =\sum_{\alpha \in \Gamma^{\prime}} W_{\alpha}\left(A P_{\alpha}\right) W_{\alpha}^{-1}=\sum_{\alpha \in \Gamma^{\prime}} \phi\left(A P_{\alpha}\right)=\phi\left(A \sum_{\alpha \in \Gamma^{\prime}} P_{\alpha}\right) \\
& =\phi(A) \phi\left(\sum_{\alpha \in \Gamma^{\prime}} P_{\alpha}\right) \\
& =\phi(A) \cdot \sum_{\alpha \in \Gamma^{\prime}} \phi\left(P_{\alpha}\right) .
\end{aligned}
$$

But $\sum_{\alpha \in \Gamma}\left(P_{\alpha}\right)=\tilde{I}=\phi(I)$; and thus by the continuity of $W(\cdot) W^{-1}, W A W^{-1}$ $=\phi(A)$.

Lemma 3.2.2. Let $\left(M, M^{\prime}\right),\left(\tilde{M}, \tilde{M}^{\prime}\right)$ be finite rings on Hilbert spaces $H$, $\tilde{H}$, with coupling operators $C, \widetilde{C}$ respectively. Further, let $z, \tilde{z}$ be vectors in $H$, $\tilde{H}$ respectively such that $\left[M^{\prime} z\right]=H,\left[\tilde{M}^{\prime} \tilde{z}\right]=\tilde{H}$. If $\phi$ is $a^{*}$-isomorphism of $M$ onto $\tilde{M}$ such that $\phi(C)=\tilde{C}$, then there exists an isometry $W$ of $H$ onto $\tilde{H}$ such that $W A W^{-1}=\phi(A)$, for $A$ in $M$.

Proof. Let $G^{\prime}=P_{[M z]}$, and, as in the previous lemma, select a projection $E^{\prime} \leqq G^{\prime}$ in $M^{\prime}$ such that $\left(E^{\prime}\right)^{\natural}=(1 / n) P$ for some projection $P$ in the center of $M$. Also, pick an orthogonal collection of projections $\left\{E_{k}^{\prime}\right\}, 1 \leqq k \leqq n$, such that each is equivalent to $E^{\prime}$ and $\sum_{k=1}^{n} E_{k}^{\prime}=I$, as well as partial isometries $V_{k}^{\prime}$ in $M^{\prime}$ with $V_{k}^{\prime *} V_{k}^{\prime}=E_{k}^{\prime}, V_{k}^{\prime} V_{k}^{\prime *}=E^{\prime}$.

Now let $E=P_{\left[M^{\prime} E^{\prime} z\right]}$ and notice that we have the equation $\left[M E^{\prime} z\right]^{\text {h }}$ $=\left(E^{\prime}[M z]\right)^{\natural}=\left(E^{\prime} G^{\prime}\right)^{\natural}=\left(E^{\prime}\right)^{\natural}=C\left[M^{\prime} E^{\prime} z\right]^{\natural}=C(E)^{\natural}$. Thus we get the result $\left(E^{\prime}\right)^{\natural}=(1 / n) P=C(E)^{\natural}$, and consequently, $(1 / n) \phi(P)=\phi(C) \cdot \phi(E)^{\natural}=\tilde{C} \cdot \phi(E)^{\natural}$. But $\left[\tilde{M}^{\prime} \phi(E) \tilde{z}\right]=\phi(E) \tilde{H}$, by hypothesis; and if we define $\tilde{E}^{\prime}=P_{[\widetilde{M} \phi(E) \bar{z}]}$, then by the definition of $\widetilde{C},\left(\tilde{E}^{\prime}\right)^{\natural}=\tilde{C} \phi(E)^{\natural}=(1 / n) \phi(P)$. Again we can choose orthogonal projections $\left\{\tilde{E}_{k}^{\prime}\right\}$ such that $\sum_{k=1}^{n} \tilde{E}_{k}^{\prime}=\phi(P)$ and with each $\tilde{E}_{k}^{\prime}$ equivalent to $\tilde{E}^{\prime}$. Let $\tilde{V}_{k}^{\prime}$ be partial isometries in $\tilde{M}^{\prime}$. such that $\tilde{V}_{k}^{\prime *} \tilde{V}_{k}^{\prime}=\tilde{E}_{k}^{\prime}$ and $\tilde{V}_{k}^{\prime} \tilde{V}_{k}^{\prime *}=\tilde{E}^{\prime}$.

But now the map $A \rightarrow A E^{\prime}$ is an isomorphism of $M P$ onto $M_{E^{\prime} H}$, since if $A E^{\prime}=0$, then, for all $k, A V_{k}^{\prime} V_{k}^{\prime *}=0=A V_{k}^{\prime} V_{k}^{\prime *} V_{k}^{\prime}=A V_{k}^{\prime}=A V_{k}^{\prime *} V_{k}^{\prime}=A E_{k}^{\prime}$ and finally $\sum_{k=1}^{n} E_{k}^{\prime} A=A P=A=0$. A similar computation shows that the mapping $\widetilde{A} \rightarrow \widetilde{A} \tilde{E}^{\prime}$ is an isomorphism of $\tilde{M} \phi(P)$ onto $\widetilde{M} \widetilde{E}^{\prime} \widetilde{H}$. We now are in a position to define the isomorphism $\phi^{\prime}$ of $M_{E^{\prime} H}$ onto $\tilde{M} \widetilde{E}_{\widetilde{H}} \widetilde{b y} \phi^{\prime}\left(A E^{\prime}\right)$ $=\phi(A) \tilde{E}^{\prime}$ for $A$ in $M P$.

Next, we observe that $\left[M_{E^{\prime} H}^{\prime} E^{\prime} z\right]=\left[E^{\prime} M^{\prime} E^{\prime} z\right]=E^{\prime}\left[M^{\prime} E^{\prime} z\right]=E E^{\prime} H$, $\left[M_{E^{\prime} H} E^{\prime} z\right]=\left[M E^{\prime} z\right]=E^{\prime}[M z]=E^{\prime} G^{\prime} H=E^{\prime} H, \quad\left[\tilde{M}^{\prime} \widetilde{E}^{\prime} \tilde{H} \phi(E) \tilde{z}\right]=\left[\tilde{E}^{\prime} \tilde{M}^{\prime} \phi(E) \tilde{z}\right]$ $=\tilde{E}^{\prime} \phi(E)\left[\tilde{M}^{\prime} \tilde{z}\right]=\tilde{E}^{\prime} \phi(E) \tilde{H},\left[\tilde{M}_{\tilde{E}^{\prime} \tilde{H}} \phi(E) \tilde{z}\right]=\tilde{E}^{\prime}[\tilde{M} \phi(E) \tilde{z}]=\tilde{E}^{\prime} \widetilde{H}$; and thus, if $C^{\prime}, \widetilde{C}^{\prime}$ are the coupling operators for the rings $\left(M_{E^{\prime} H}, M_{E^{\prime} H}^{\prime}\right),\left(\tilde{M}_{\widetilde{E}^{\prime} \widetilde{H}}, \tilde{M}_{\widetilde{E}^{\prime} \widetilde{H}}^{\prime}\right)$ respectively and if the symbol ${ }^{\circ}$ denotes the trace in the above reduced rings, then $\left(E^{\prime}\right)^{\circ}=C^{\prime}\left(E E^{\prime}\right)^{\circ}$ and $\left(\tilde{E}^{\prime}\right)^{\circ}=\tilde{C}^{\prime}\left(\tilde{E}^{\prime} \phi(E)\right)^{\circ}$. But this implies that $\phi^{\prime}\left(E^{\prime}\right)^{\circ}$ $=\tilde{C}^{\prime} \phi^{\prime}\left(E E^{\prime}\right)^{\circ}=\phi^{\prime}\left(C^{\prime}\right) \cdot \phi^{\prime}\left(E E^{\prime}\right)^{\circ}$ and proves that $\phi^{\prime}\left(C^{\prime}\right)=\tilde{C}^{\prime}$. This fact, plus 
Lemma 3.2.1, yields a linear isometry $W_{0}$ of $E^{\prime} H$ onto $\tilde{E}^{\prime} \tilde{H}$ such that $\phi^{\prime}\left(A E^{\prime}\right)$ $=W_{0} A E^{\prime} W_{0}^{-1}$.

As in the proof of Lemma 3.2.1, we can define a linear isometry from $P H$ onto $\phi(P) \tilde{H}$ such that $W=\sum_{k=1}^{n} \widetilde{V}_{k}^{\prime} * W_{0} V_{k}^{\prime}$ and compute (for $A$ in $M P$ ),

$$
\begin{aligned}
W A W^{-1} & =\sum_{k, s=1}^{n} \tilde{V}_{k}^{\prime *} W_{0} V_{k}^{\prime} A V_{s}^{\prime *} W_{0}^{-1} \tilde{V}_{s}^{\prime} \\
& \left.=\sum_{k=1}^{n} \tilde{V}_{k}^{\prime *} W_{0}\left(V_{k}^{\prime} V_{k}^{\prime *} A\right) W_{0}^{-1} \tilde{V}_{k}^{\prime} \quad \text { (since } V_{k}^{\prime} V_{s}^{\prime *}=0 \text { for } k \neq s\right) \\
& =\sum_{k=1}^{n} \tilde{V}_{k}^{\prime *} W_{0}\left(E^{\prime} A\right) W_{0}^{-1} \tilde{V}_{k}^{\prime}=\sum_{k=1}^{n} \tilde{V}_{k}^{\prime *} \cdot \phi^{\prime}\left(E^{\prime} A\right) \tilde{V}_{k}^{\prime} \\
& =\sum_{k=1}^{n} \tilde{V}_{k}^{\prime *} \tilde{E}^{\prime} \phi(A) \tilde{V}_{k}^{\prime}=\sum_{k=1}^{n} \tilde{V}_{k}^{\prime *} \tilde{V}_{k}^{\prime} \phi(A)=\sum_{k=1}^{n} \tilde{E}_{k}^{\prime} \phi(A) \\
& =\phi(P) \phi(A)=\phi(A P)=\phi(A) .
\end{aligned}
$$

Thus we have proved that there is a linear isometry implementing $\phi$ on $M P$, for some projection $P$ in the center of $M$. The remainder of the proof is identical with the last part of the proof of Lemma 3.2.1.

LEMma 3.2.3. Let $\left(M, M^{\prime}\right),\left(\tilde{M}, \tilde{M}^{\prime}\right)$ be finite rings on Hilbert spaces $H, \tilde{H}$, with coupling operators $C, \tilde{C}$ respectively. If $\phi$ is a ${ }^{*}$-isomorphism of $M$ onto $\tilde{M}$ such that $\phi(C)=\tilde{C}$, then there exists a linear isometry $W$ of $H$ onto $\tilde{H}$ such that $W A W^{-1}=\phi(A)$ for $A$ in $M$.

Proof. By Definition 3.1 and the proof of Theorem 1, we have collections of orthogonal projections $\left\{P_{\alpha}\right\},\left\{E_{\alpha}\right\},\left\{\tilde{P}_{\alpha}\right\},\left\{\tilde{E}_{\alpha}\right\}(\alpha \in \Gamma)$ in the center of $M, M P_{\alpha}, \tilde{M}, \tilde{M} \tilde{P}_{\alpha}$ respectively with vectors $x_{\alpha, 1}, x_{\alpha, 2}, \tilde{x}_{\alpha, 1}, \tilde{x}_{\alpha, 2}$ in $H, \tilde{H}$ such that $\sum_{\alpha \in \Gamma} \tilde{P}_{\alpha}=\tilde{I}$,

$$
\begin{aligned}
{\left[M x_{\alpha, 1}\right] } & =E_{\alpha} P_{\alpha} H, & {\left[M^{\prime} x_{\alpha, 1}\right]^{\natural} } & =D_{\alpha^{\prime}} \\
{\left[M x_{\alpha, 2}\right]^{\natural} } & =C_{\alpha}, & {\left[M^{\prime} x_{\alpha, 2}\right] } & =E^{\perp} P_{\alpha} H, \\
{\left[\tilde{M} \tilde{x}_{\alpha, 1}\right] } & =\tilde{E}_{\alpha} \tilde{P}_{\alpha} \tilde{H}, & {\left[\tilde{M}^{\prime} \tilde{x}_{\alpha, 1}\right]^{\natural} } & =\widetilde{D}_{\alpha}, \\
{\left[\tilde{M} \tilde{x}_{\alpha, 2}\right]^{\natural} } & =\tilde{C}_{\alpha}, & {\left[\tilde{M}^{\prime} \tilde{x}_{\alpha, 2}\right] } & =\tilde{E}_{\alpha}^{\perp} \tilde{P}_{\alpha} \tilde{H},
\end{aligned}
$$

and finally

$$
\phi\left(C_{\alpha}\right)=\tilde{C}_{\alpha}, \quad \phi\left(D_{\alpha}^{-1}\right)=\tilde{D}_{\alpha}^{-1} .
$$

Clearly, $\phi\left(P_{\alpha}\right)=\tilde{P}_{\alpha}, \phi\left(E_{\alpha}\right)=\tilde{E}_{\alpha}$ and we can apply Lemmas 3.2.1 and 3.2.2 to get linear isometries $W_{\alpha, k}, \alpha \in \Gamma, k=1,2$, implementing $\phi$ on the rings $M E_{\alpha} P_{\alpha}, M E_{\alpha}^{\perp} P_{\alpha}$. Having done this, we get an isometry $W$ in the large by the method of Lemma 3.2.1, thus completing the proof of this lemma.

Lemma 3.2.4. Let $M, M^{\prime}$ be of types $S_{1}, S_{\Omega}(\Omega$ infinite) respectively. There 
exists a collection of orthogonal, equivalent, finite projections $\left\{E_{\alpha}^{\prime}\right\}, \alpha \in \Gamma$, such that $\sum_{\alpha \in \Gamma} E_{\alpha}^{\prime}=I$; the $M_{E^{\prime} H}$ are all isomorphic to $M$, and the coupling operator for $\left(M_{E_{\alpha^{\prime} H}}, M_{E_{\alpha^{\prime} H}}^{\prime}\right)$ is the identity.

Proof. We first consider the case when there is a vector $x$ such that $\left[M^{\prime} x\right]$ $=H$. The projection $F_{0}^{\prime}=P_{[M z]}$ is finite by Lemma 1.2.4. Now, by Zorn's Lemma, pick a maximal family $\left\{F_{\alpha}^{\prime}\right\}, \alpha \in \Gamma$, of orthogonal projections equivalent to $F_{0}^{\prime}$ and let $F^{\prime}=\sum_{\alpha \in \Gamma} F_{\alpha}^{\prime}$. Comparing $F_{0}^{\prime}$ with $\left(F^{\prime}\right)^{\perp}$, we notice that $F_{0}^{\prime} \prec\left(F^{\prime}\right)^{\perp}$ is false by the maximality of our family $\left\{F_{\alpha}^{\prime}\right\}$; and thus there exists a projection $P$ in the center of $M$ such that $P\left(F^{\prime}\right)^{\perp} \prec P F_{0}^{\prime}$ (proper).

We now have $P=\sum_{\alpha \in \Gamma} P F_{\alpha}^{\prime}+P\left(F^{\prime}\right)^{\perp}$ and the set $\Gamma$ is clearly of infinite cardinality (by finiteness of $\left.F_{0}^{\prime}\right)$. Further, the projections $P F_{\alpha}^{\prime}$ are all equivalent to $P F_{0}^{\prime}$. Let $\alpha_{0}$ be any element of $\Gamma$ and consider the fact that $\sum_{\alpha \in \Gamma-\alpha_{0}} P F_{\alpha}^{\prime} \sim \sum_{\alpha \in \Gamma} P F_{\alpha}^{\prime}$ as each of the index sets has the same cardinality. But this obviously leads to the equation $\sum_{\alpha \in \Gamma} P F_{\alpha}^{\prime}>\sum_{\alpha \in \Gamma-\alpha_{0}} P F_{\alpha}^{\prime}$ $+P\left(F^{\prime}\right)^{\perp}>\sum_{\alpha \in \Gamma} P F_{\alpha}^{\prime}+P\left(F^{\prime}\right)^{\perp}=P$ or $P \sim \sum_{\alpha \in \Gamma} P F_{\alpha}^{\prime}$.

Next, select a partial isometry $V^{\prime}$ in $M^{\prime}$ such that $V^{*} V^{\prime}=P$ and $V^{\prime} V^{\prime *}=\sum_{\alpha \in \Gamma} P F_{\alpha}^{\prime}$. Then, $P=V^{\prime *} V^{\prime} V^{\prime *} V^{\prime}=V^{\prime *}\left(\sum_{\alpha \in \Gamma} P F_{\alpha}^{\prime}\right) V^{\prime}$ $=\sum_{\alpha \in \Gamma} P V^{\prime *} F_{\alpha}^{\prime} V^{\prime}$, where the $P V^{\prime *} F_{\alpha}^{\prime} V^{\prime}$ are clearly equivalent to $P F_{0}^{\prime}$. Thus we have expressed a central projection $P$ as a sum of orthogonal projections equivalent to $P F_{0}^{\prime}$.

Again apply Zorn's Lemma to obtain a maximal family of orthogonal projections $\left\{P_{\alpha}\right\}, \alpha \in \Lambda$, such that $P_{\alpha}=\sum_{\gamma \in \Gamma} F_{\alpha, \gamma}^{\prime}$, the $F_{\alpha, \gamma}^{\prime}$ being orthogonal projections equivalent to $P_{\alpha} F_{0}^{\prime}$ (the same index set $\Gamma$ can be used for the $F_{\alpha, \gamma}^{\prime}$ since all have the cardinal $\Omega$ ). Form $P=\sum_{\alpha \in \Delta} P_{\alpha}$, and consider $P^{\perp}$. If $P^{\perp} \neq 0$, then the process described in the first part of the proof of this lemma shows that there is a nonzero projection in the center of $M\left(P^{\perp}\right)$ which is a sum of projections equivalent to $F_{0}^{\prime}$ cut down to that central projection. This contradiction of maximality of the family $\left\{P_{\alpha}\right\}$ proves that $P=I$.

Now, form the projections $E_{\gamma}=\sum_{\alpha \in \Lambda} F_{\alpha, \gamma}^{\prime}$. These projections are obviously equivalent to $\sum_{\alpha \in \Delta} P_{\alpha} F_{0}^{\prime}=F_{0}^{\prime}$ and $I=\sum_{\gamma \in \Gamma} E_{\gamma}^{\prime}$. Furthermore, the rings $M_{E^{\prime} H}$ are isomorphic to $M_{F_{0}{ }^{\prime} H}$. Also, the mapping $A \rightarrow A F_{0}^{\prime}$ is an isomorphism of $M$ onto $M_{F_{0}{ }^{\prime} H}$, since if $A F_{0}^{\prime}=0$, then $A x=0$ and hence $A=0$. Finally, $\left[M_{F_{0^{\prime}} H} x\right]=\left[M_{F_{0^{\prime} H}}^{\prime} x\right]=F_{0}^{\prime} H$ shows that the coupling operator for the rings $M_{F_{0^{\prime} H},}, M_{F_{0}{ }^{\prime} H}^{\prime}$ is the identity. This completes the proof of the case when $\left[M^{\prime} x\right]=H$ for some $x$ in $H$.

In the general case, we use Lemmas 1.2.7 and 1.2.8 to obtain a family $\left\{P_{\alpha}\right\}, \alpha \in \Lambda$, of orthogonal projections in the center of $M$ such that $\sum_{\alpha \in \Delta} P_{\alpha}$ $=I$ and with either $\left[M x_{\alpha}\right]=P_{\alpha} H$ or $\left[M^{\prime} x_{\alpha}\right]=P_{\alpha} H$ for vectors $x_{\alpha}$ in $H$. But if $\left[M x_{\alpha}\right]=P_{\alpha} H$ for some $\alpha \in \Lambda$, then, by Lemma 1.2.4, $\left[M x_{\alpha}\right]$ and thus $P_{\alpha}$ would be finite in $M^{\prime}$, contradicting the fact that $M^{\prime}$ is of type $S_{\Omega}$. Thus all the $P_{\alpha}$ satisfy $\left[M^{\prime} x_{\alpha}\right]=P_{\alpha} H$. 
Applying our previous results to the rings $M P_{\alpha}$, we obtain a set of equivalent, finite, orthogonal projections $E_{\alpha, \gamma}^{\prime}, \alpha \in \Lambda, \gamma \in \Gamma$, such that $P_{\alpha}$ $=\sum_{\gamma \in \Gamma} E_{\alpha, \gamma}^{\prime}$ and the rings $M P_{\alpha E^{\prime} \alpha, \gamma^{\prime} H}, M^{\prime} P_{\alpha E^{\prime} \alpha, \gamma^{H}}$ have the identity as a coupling operator. But finally, defining $E_{\gamma}^{\prime}=\sum_{\alpha \in \Lambda} E_{\alpha, \gamma}^{\prime}$, we see that the $E_{\gamma}^{\prime}$ are a family of orthogonal, equivalent, finite projections with $\sum_{\gamma} \in \Gamma E_{\gamma}^{\prime}$ $=I$. Since $P_{\alpha} E_{\gamma}^{\prime}=E_{\alpha, \gamma}^{\prime}, \quad\left(M P_{\alpha E_{\gamma^{\prime} H}}, \quad M^{\prime} P_{\alpha E_{\gamma^{\prime} H}}\right)=\left(M P_{\alpha E^{\prime} \alpha, \gamma H}, M^{\prime} P_{\alpha E^{\prime} \alpha, \gamma H}\right)$; thus the coupling operator of $\left(M_{E_{\gamma^{\prime} H}}, M_{E_{\gamma^{\prime} H} H}^{\prime}\right)$ on $P_{\alpha} E_{\gamma}$ is the identity. But piecing together the coupling operators on $P_{\alpha} E_{\gamma}^{\prime}$ we obtain finally that the coupling operator of $\left(M_{E_{\gamma^{\prime} H}}, M_{E_{\gamma^{\prime} H}}^{\prime}\right)$ is the identity.

To complete the proof of this lemma, notice that the mapping $A \rightarrow A E_{\gamma}^{\prime}$ is a ${ }^{*}$-isomorphism of $M$ onto $M_{E_{\gamma^{\prime} H}}$; since if $A E_{\gamma}^{\prime}=0, A P_{\alpha} E_{\gamma}^{\prime}=0$ which yields $A P_{\alpha}=0$ for all $\alpha$, by the first part of this proof. Then, $A=0$ follows from $\sum_{\alpha \in \Lambda} P_{\alpha}=I$.

Lemma 3.2.5. Let $M, M^{\prime}, \widetilde{M}, \tilde{M}^{\prime}$, be rings of types $S_{1}, S_{\Omega}, S_{1}, S_{\Omega}$ respectively ( $\Omega$ an infinite cardinal). If $\phi$ is $a^{*}$-isomorphism of $M$ onto $\tilde{M}$, then there exists a linear isometry $W$ mapping $H$ onto $\widetilde{H}$, such that $\phi(A)=W A W^{-1}$ for $A$ in $M$.

Proof. Applying the preceding lemma to the above rings, we get the families $\left\{E_{\alpha}^{\prime}\right\},\left\{\tilde{E}_{\alpha}^{\prime}\right\}, \alpha \in \Gamma$, of $\Omega$ equivalent, finite, orthogonal projections in $M^{\prime}, \tilde{M}^{\prime}$ respectively such that $\sum_{\alpha \in \Gamma} E_{\alpha}^{\prime}=I, \sum_{\alpha \in \Gamma} \tilde{E}_{\alpha}^{\prime}=\tilde{I}$ and such that the coupling operators of the rings $\left(M_{E_{\alpha^{\prime}} H}, M_{E_{\alpha^{\prime} H}}^{\prime}\right),\left(\widetilde{M}_{\widetilde{E}_{\alpha^{\prime}}} \widetilde{H}^{\prime}, \tilde{M}_{\boldsymbol{E}_{\alpha^{\prime}} \tilde{H}^{\prime}}^{\prime}\right)$ are the identity operators. Further, one can define the ${ }^{*}$-isomorphism $\phi^{\prime}$ of $M_{E \alpha_{0}{ }^{\prime} H}$ onto $\widetilde{M}_{\widetilde{E}_{\alpha_{0}}{ }^{\prime} \widetilde{H}^{\prime}}$ by $\phi^{\prime}\left(A E_{\alpha_{0}}^{\prime}\right)=\phi(A) \tilde{E}_{\alpha_{0}}^{\prime}$ for $A$ in $M$. Lemma 3.2.3 can now be invoked to yield a linear isometry $W_{0}$ of $E_{\alpha_{0}}^{\prime} H$ onto $\tilde{E}_{\alpha_{0}}^{\prime} \tilde{H}$ such that $W_{0}\left(A E_{\alpha_{0}}^{\prime}\right) W_{0}^{-1}=\phi(A) \tilde{E}_{\alpha_{0}}^{\prime}$.

Pick partial isometries $\left\{V_{\alpha}^{\prime}\right\},\left\{\tilde{V}_{\alpha}^{\prime}\right\}, \alpha \in \Gamma$, in $M^{\prime}, \tilde{M}^{\prime}$ respectively such that $V_{\alpha}^{\prime *} V_{\alpha}^{\prime}=E_{\alpha}^{\prime}, V_{\alpha}^{\prime} V_{\alpha}^{\prime *}=E_{\alpha 0}^{\prime}, \tilde{V}_{\alpha}^{\prime} * \tilde{V}_{\alpha}^{\prime}=\widetilde{E}_{\alpha}^{\prime}, \tilde{V}_{\alpha}^{\prime} \tilde{V}_{\alpha}^{\prime *}=\tilde{E}_{\alpha_{0}}^{\prime}$. As in the proof of Lemma 3.2.2, we define the linear isometry $W=\sum_{\alpha \in \Gamma} \widetilde{V}_{\alpha}^{\prime *} W_{0} V_{\alpha}^{\prime}$ of $H$ onto $\widetilde{H}$; and, if $\Gamma^{\prime}$ is any finite subset of $\Gamma$ (by the same computation as in Lemma 3.2.2), $\left(\sum_{\alpha \in \Gamma^{\prime}} \tilde{V}_{\alpha}^{\prime *} W_{0} V_{\alpha}^{\prime}\right) A\left(\sum_{\alpha \in \Gamma^{\prime}} V_{\alpha}^{\prime *} W_{0}^{-1} \tilde{V}_{\alpha}^{\prime}\right)=\phi(A)\left(\sum_{\alpha \in \Gamma^{\prime}} \tilde{E}_{\alpha}^{\prime}\right)$ $=W \sum_{\alpha \in \Gamma^{\prime}} E_{\alpha}^{\prime} A W^{-1}$. Thus by continuity of $W, W A W^{-1}=\phi(A)$.

Lemma 3.2.6. Let $M, M^{\prime}, \widetilde{M}, \tilde{M}^{\prime}$ be rings of types $S_{\Omega}, S_{\Omega^{\prime}}, S_{\Omega}, S_{\Omega^{\prime}}$ respectively ( $\Omega, \Omega^{\prime}$ being infinite cardinals). If $\phi$ is a ${ }^{*}$-isomorphism of $M$ onto $\tilde{M}$, then there exists a linear isometry $W$ mapping $H$ onto $\widetilde{H}$, such that $\phi(A)$ $=W A W^{-1}$ for $A$ in $M$.

Proof. Since $M$ is of type $S_{\Omega}$, there exists a family $\left\{E_{\alpha}\right\}, \alpha \in \Gamma$, of $\Omega$ orthogonal, finite, equivalent projections in $M$ such that $\sum_{\alpha \in \Gamma} E_{\alpha}=I$. If we let $\phi\left(E_{\alpha}\right)=\tilde{E}_{\alpha}$, the family $\left\{\tilde{E}_{\alpha}\right\}, \alpha \in \Gamma$, is a family of projections in $\tilde{M}$ with properties similar to those of $\left\{E_{\alpha}\right\}$. For $\alpha_{0}$ a particular element of $\Gamma$, there exist partial isometries $\left\{V_{\alpha}\right\}, \alpha \in \Gamma$, in $M$ such that $V_{\alpha}^{*} V_{\alpha}=E_{\alpha}, V_{\alpha} V_{\alpha}^{*}$ $=E_{\alpha_{0}}$.

We notice that $\phi$ induces a ${ }^{*}$-isomorphism of $M_{E_{\alpha_{0} H}}$ onto $\widetilde{M}_{\widetilde{E}_{\alpha} \widetilde{H}}$. Further, the map $A^{\prime} \rightarrow A^{\prime} E_{\alpha_{0}}$ is an isomorphism of $M^{\prime}$ onto $M_{E_{\alpha} H}^{\prime}$; since if $A^{\prime} E_{\alpha_{0}}=0$, 
then by the same argument as the third paragraph of the proof of Lemma 3.2.2, this implies $A^{\prime} E_{\alpha}=0$ for all $\alpha \in \Gamma$ and hence $A^{\prime}$ would vanish. A similar argument shows that $\tilde{M}^{\prime} \widetilde{E}_{\alpha_{0} \widetilde{H}}$ is isomorphic to $\tilde{M}^{\prime}$. Thus the rings $M_{E_{\alpha_{0} H}}$, $M_{E_{\alpha_{0} H} H}^{\prime}, \widetilde{M}_{\widetilde{E}_{\alpha_{0}} \widetilde{H}}, \widetilde{M}^{\prime} \widetilde{E}_{\alpha_{0}} \widetilde{H}$ are of types $S_{1}, S_{\Omega^{\prime}}, S_{1}, S_{\Omega^{\prime}}$, and we can apply Lemma 3.2 .5 to get a linear isometry $W_{0}$ with the property: $W_{0} A W_{0}^{-1}=(A)$ for $A$ in the ring $M_{E_{\alpha_{0}} H}$.

Now, we can define the linear isometry $W=\sum_{\alpha \in \Gamma} \phi\left(V_{\alpha}^{*}\right) W_{0} V_{\alpha}$, and if $\Gamma^{\prime}$ is any finite subset of $\Gamma$ then, by the same computation as that of the third paragraph of the proof of Lemma 3.2.1,

$$
W\left(\sum_{\alpha \in \Gamma^{\prime}} E_{\alpha}\right) A\left(\sum_{\alpha \in \Gamma^{\prime}} E_{\alpha}\right) W^{-1}=\sum_{\alpha, \beta \in \Gamma^{\prime}}\left(E_{\alpha} A E_{\beta}\right)=\sum_{\alpha \in \Gamma^{\prime}}\left(E_{\alpha}\right)(A) \sum_{\beta \in \Gamma^{\prime}}\left(E_{\beta}\right) .
$$

But now, by the continuity properties of $W$, we get finally: $\phi(A)=W A W^{-1}$.

Definition 3.2. Let $M, M^{\prime}, \tilde{M}, \tilde{M}^{\prime}$ be semifinite rings of operators on Hilbert spaces $H, \tilde{H}$, with coupling operators $C, \widetilde{C}$ respectively. If, as in Definition 1.6, we write

$$
C=C_{1}+\sum_{\alpha, \alpha^{\prime} \neq 1,1} \frac{\alpha}{\alpha^{\prime}} P_{\alpha, \alpha^{\prime}}, \quad \tilde{C}=\tilde{C}_{1}+\sum_{\alpha, \alpha^{\prime} \neq 1,1} \frac{\alpha}{\alpha^{\prime}} \tilde{P}_{\alpha, \alpha^{\prime}},
$$

then a *-isomorphism $\phi$ mapping $M$ onto $\tilde{M}$ is said to take $C$ into $\tilde{C}$, if $\phi\left(P_{\alpha, \alpha^{\prime}}\right)=\widetilde{P}_{\alpha, \alpha^{\prime}}$ for all $\alpha, \alpha^{\prime}$ and if $\phi\left(C_{1}\right)=\widetilde{C}_{1} . C$ is said to be essentially bounded if all the $\left\{P_{\alpha, 1}\right\}, \alpha>1$, are zero.

TheOREM 9. Let $M, M^{\prime}, \tilde{M}, \tilde{M}^{\prime}$ be semifinite rings of operators on Hilbert spaces $H, \tilde{H}$, with essentially bounded coupling operators $C, \tilde{C}$ respectively. If $\phi$ is $a^{*}$-isomorphism of $M$ onto $\tilde{M}$ taking $C$ into $\tilde{C}$, then there exists a linear isometry $W$ mapping $H$ onto $\tilde{H}$ such that $\phi(A)=W A W^{-1}$ for $A$ in $M$.

Proof. Let $C=C_{1}+\sum\left(\alpha / \alpha^{\prime}\right) P_{\alpha, \alpha^{\prime}}$. By Lemma 3.2.3, there exists a linear isometry implementing $\phi$ on $P_{1,1}$. By Lemma 3.2.5, there exists a linear isometry implementing $\phi$ on the $P_{1, \alpha}, \alpha>1$; and by Lemma 3.2.6, there is a linear isometry implementing $\phi$ on the $P_{\alpha, \alpha^{\prime}}$ for $\alpha, \alpha^{\prime}>1$.

Thus we have a collection of orthogonal projections in the center of $M$ such that on each one $\phi$ is implemented by a linear isometry. But now by the argument at the end of the proof of Lemma 3.2.1, there exists a linear isometry rmplementing $\phi$ in the large. This completes the proof of the theorem.

THEOREM 10. Let $M, M^{\prime}$ be semifinite rings with an essentially bounded coupling operator $C$. If $\phi$ is $a^{*}$-automorphism of $M$ leaving $C$ fixed, then there exists a unitary operator $W$ on the Hilbert space $H$ such that $\phi(A)=W A W^{*}$ for $A$ in $M$.

Proof. $\phi$ is clearly a *-isomorphism of $M$ onto itself, which takes the 
coupling operator $C$ into the coupling operator of its image. Thus we can apply Theorem 9, obtaining a linear isometry $W$ of $H$ onto itself such that $\phi(A)=W A W^{-1}$ for $A$ in $M$. But such a $W$ is unitary on $H$, and consequently $W^{-1}=W^{*}$.

\subsection{Continuity properties of *-isomorphisms.}

Lemma 3.3.1. Let $M$ be a semifinite ring on the Hilbert space $H$. If $\pi$ and $\left\{P_{\alpha}\right\}$ are the cardinals, projections respectively of Theorem 3 , let $\Omega$ be an infinite cardinal larger than any of the cardinals in $\pi$. If $\Gamma$ is an abstract set of cardinal $\Omega$, then the ring $\tilde{M}$ of Theorem 4 is of type $S_{\Omega}$.

Proof. Let $\phi$ be the isomorphism of $M^{\prime}$ onto $\tilde{M}^{\prime}$ given in Theorem 4. With the notation of the proof of that theorem, consider the family $\phi\left(P_{\alpha}\right) e_{\gamma}, \gamma \in \Gamma$ ( $\alpha$ fixed). These projections are clearly all equivalent, and since the $\tilde{M}_{e_{\gamma} \tilde{H}}$ are unitarily equivalent to $M$, each $\phi\left(P_{\alpha}\right) e_{\gamma}$ of the above family is the sum of $\alpha$ orthogonal, equivalent, finite projections in $\tilde{M}$. Thus $\phi\left(P_{\alpha}\right)$ is the sum of $\Omega \cdot \alpha=\Omega$ such projections, and $\tilde{M} \phi\left(P_{\alpha}\right)$ is of type $S_{\Omega}$.

But $\sum_{\alpha \in \pi} \phi\left(P_{\alpha}\right)=\phi(I)=I$ proves that $\tilde{M}$ is of type $S_{\Omega}$, and the proof of this lemma is complete.

THEOREM 11. The strongest and $\sigma$-weak topologies are purely algebraic in semifinite rings; that is, any *-isomorphism between semifinite rings is bicontinuous in the strongest and $\sigma$-weak topologies.

Proof. Let $\phi$ be a ${ }^{*}$-isomorphism of the semifinite ring $M_{1}$ onto $M_{2}$. Pick an abstract set $\Gamma$ with infinite cardinality $\Omega$ larger than that of the Hilbert spaces on which the rings $M_{k}, k=1,2$, act, and apply the process outlined in Theorem 4 to the rings $M_{k}^{\prime}$. Let $\phi_{1}, \phi_{2}$ be the isomorphisms of $M_{1}, M_{2}$ onto $\tilde{M}_{1}$. $\tilde{M}_{2}$ respectively, and form the isomorphism $\tilde{\phi}=\phi_{2} \phi \phi_{1}^{-1}$.

$\tilde{\phi}$ is now a ${ }^{*}$-isomorphism of $\widetilde{M}_{1}$ onto $\tilde{M}_{2}$. In $\tilde{M}_{1}$ form the family $\left\{\tilde{P}_{\alpha}\right\}$, as in Theorem 3. But, from our selection of $\Omega$, and by Lemma 3.3.1, it is clear that the $\tilde{M}_{k}^{\prime}$ are of type $S_{\Omega}$, and thus our coupling operator $\tilde{C}_{1}$ for $\tilde{M}_{1}$ has the form: $\tilde{C}_{1}=\sum_{\alpha \in \pi_{1}}(\alpha / \Omega) \tilde{P}_{\alpha}$. Since the types $S_{\alpha}$ are preserved under ${ }^{*}$-isomorphism, we also get the coupling operator $\tilde{C}_{2}$ of $\tilde{M}_{2}$ in the form: $\tilde{C}_{2}$ $=\sum_{\alpha \in \pi_{1}}(\alpha / \Omega) \tilde{\phi}\left(\tilde{P}_{\alpha}\right) \dot{\tilde{\phi}}$

Thus we see that $\tilde{\phi}$ takes $\tilde{C}_{1}$ into $\tilde{C}_{2}$, with each being essentially bounded. Applying Theorem 9, we see that there exists a linear isometry implementing $\tilde{\phi}$. But now $\tilde{\phi}$ is obviously bicontinuous in both the strongest and $\sigma$-weak topologies; and since the $\phi_{k}, k=1,2$, are bicontinuous in these topologies by Theorem 4, we get finally the result that $\phi=\phi_{2}^{-1} \tilde{\phi} \phi_{1}$ is bicontinuous in the required topologies. This completes the proof of the theorem.

Lemma 3.3.2. If $M, M^{\prime}$ are purely infinite semifinite rings on a Hilbert space $H$, then the strongest and strong topologies are equivalent and the $\sigma$-weak and weak topologies are equivalent. 
Proof. Let $\Gamma$ be the set of positive integers, and apply Theorem 4 to $M^{\prime}$, obtaining rings $\tilde{M}, \tilde{M}^{\prime}$ and an isomorphism $\phi$ of $M$ onto $\tilde{M}$. If the projections $\left\{P_{\alpha, \alpha^{\prime}}\right\}, \alpha, \alpha^{\prime} \in \pi$, are the ones in the standard decomposition of the coupling operator for $\left(M, M^{\prime}\right)$, consider the rings $\phi\left(P_{\alpha, \alpha^{\prime}}\right) \tilde{M}, \phi\left(P_{\alpha, \alpha^{\prime}}\right) \tilde{M}^{\prime}$. As $\phi$ is an isomorphism, the ring $\phi\left(P_{\alpha, \alpha^{\prime}}\right) \tilde{M}$ is of type $S_{\alpha}$ like $P_{\alpha, \alpha^{\prime}} M$. Also, as in the proof of Lemma 3.3.1, the family $\phi\left(P_{\alpha, \alpha^{\prime}}\right) e_{n}, n \in \Gamma$ ( $\alpha$ fixed), are all equivalent; and as each member of that family is the sum of $\alpha^{\prime}$ orthogonal, equivalent, finite projections, we get finally that $\phi\left(P_{\alpha, \alpha^{\prime}}\right)$ is the sum of $\alpha^{\prime} \cdot \boldsymbol{\aleph}_{0}=\alpha^{\prime}$ such projections. This proves that $\phi\left(P_{\alpha, \alpha^{\prime}}\right) \tilde{M}^{\prime}$ is of type $S_{\alpha^{\prime}}$.

Thus we see that if $\left\{P_{\alpha, \alpha^{\prime}}\right\}, \alpha, \alpha^{\prime} \in \pi$, is a decomposition of the coupling operator of $\left(M, M^{\prime}\right)$, then $\phi\left(P_{\alpha, \alpha^{\prime}}\right)$ is the corresponding decomposition of the coupling operator of $\left(\tilde{M}, \tilde{M}^{\prime}\right)$. Since both operators are obviously essentially bounded, we may apply Theorem 9 to obtain a linear isometry $W$ implementing $\phi$.

Now let $\left\{x_{n}\right\}$ be a sequence of vectors in $H$ such that $\sum_{n=1}^{\infty}\left\|x_{n}\right\|^{2}<\infty$, and, using the notation of Theorem 4, define $\tilde{x}_{n}=V_{n} x_{n}$. Clearly we may define a vector $\tilde{x}=\sum_{n=1}^{\infty} \tilde{x}_{n}$ in $\tilde{H}$; and, by the proof of Theorem 4 , for $A$ in $M, \sum_{n=1}^{\infty}\left(A V_{n}^{-1} \tilde{x}_{n}, V_{n}^{-1} \tilde{x}_{n}\right)=\sum_{n=1}^{\infty}\left(A x_{n}, x_{n}\right)=(\phi(A) \bar{x}, \tilde{x})$ $=\left(W A W^{-1} \tilde{x}, \tilde{x}\right)$, or $\sum_{n=1}^{\infty}\left(A x_{n}, x_{n}\right)=\left(A W^{-1} \tilde{x}, W^{-1} \tilde{x}\right)$. That the strongest and the strong, the weak and the $\sigma$-weak topologies are equivalent is now clear.

THEOREM 12. Let $M, M^{\prime}$ be semifinite rings on a Hilbert space $H$, with coupling operator $C$. If $C$ is bounded, the strongest and the strong, the weak and the $\sigma$-weak topologies are equivalent pairs of topologies.

Proof. Let $C=C_{1}+\sum\left(\alpha / \alpha^{\prime}\right) P_{\alpha, \alpha^{\prime}}$ be the standard decomposition of the coupling operator. By boundedness of $C$, the $P_{\alpha, 1}, \alpha>1$, are all zero and $C_{1}$ is a bounded operator. Thus $Q^{\perp}=\sum_{\left(\alpha, \alpha^{\prime}\right) \neq 1} P_{\alpha, \alpha^{\prime}}$ has the property that $M\left(Q^{\perp}\right)$ and $M^{\prime}\left(Q^{\perp}\right)$ are purely infinite semifinite rings, and we see that the theorem is true on $(Q)^{\perp} H$, by Lemma 3.3.2.

Now, let $\left\{x_{n}\right\}$ be a sequence of vectors in $Q H$ such that $\sum_{n=1}^{\infty}\left\|x_{n}\right\|^{2}<\infty$, and form the linear functional $\omega(A)=\sum_{n=1}^{\infty}\left(A x_{n}, x_{n}\right)$ on $M Q$. This functional clearly satisfies all the conditions of Theorem 7 , and thus there exists a finite set $\left\{z_{k}\right\}, 1 \leqq k \leqq m$, with $\omega(A)=\sum_{k=1}^{m}\left(A z_{k}, z_{k}\right)$. But this proves that the theorem is true on $Q H$. Combining this result with that of the first paragraph, we finally complete the proof.

THEOREM 13. The trace function in a finite ring is continuous in the strongest and $\sigma$-weak topologies.

Proof. Let $M$ be the finite ring, and with $\Gamma$ as the positive integers, apply Theorem 4 to $M^{\prime}$, getting rings $\widetilde{M}, \tilde{M}^{\prime}$ and an isomorphism $\phi$ of $M$ onto $\tilde{M}$. Since $\tilde{M}^{\prime}$ is purely infinite, the coupling operator for $\left(\tilde{M}, \tilde{M}^{\prime}\right)$ satisfies the conditions of Theorem 12, proving that the strong and the strongest, the weak and $\sigma$-weak topologies are equivalent pairs of topologies on $\tilde{M}$. 
Now, let $\left\{A_{\alpha}\right\}$ be a directed set of operators in $M$ which converge to zero in the strongest topology. By the continuity properties of $\phi, \phi\left(A_{\alpha}\right)$ converges to zero in the strongest (and thus the strong) topology on $\tilde{M}$. Again using the fact that $\tilde{M}^{\prime}$ is purely infinite, Theorem 8 can be applied, yielding $\phi\left(A_{\alpha}\right)^{\text {q }}$ $\rightarrow 0$ in the strong (and thus the strongest) topology. Then, applying $\phi^{-1}$, we get $A_{\alpha}^{\natural}$ converging to zero in the strongest topology. This proves that the trace is continuous in the strongest topology, and a similar argument does the same for the $\sigma$-weak topology.

THEOREM 14. The notion of subring is purely algebraic with respect to semifinite rings; that is, if $\phi$ is a ${ }^{*}$-isomorphism of the semifinite ring $M$ onto the ring $\tilde{M}$ and if $N$ is a semifinite subring of $M$, then $\phi(N)$ is a subring of $\tilde{M}$.

Proof. If $\tilde{N}$ is defined as the strongest closure of $\phi(N)$, it is clearly sufficient for the proof of the theorem to prove that $\tilde{N} \subseteq \phi(N)$. Therefore, let $\tilde{A}$ be in $\tilde{N}$ and pick a directed set $\left\{\tilde{A}_{\alpha}\right\}$ of operators in $\phi(N)$ converging to $\tilde{A}$ in the strongest topology. But by Theorem $11, \phi^{-1}$ is strongest continuous from $\widetilde{M}$ to $M$, so that we have the directed set $\phi^{-1}\left(\widetilde{A}_{\alpha}\right)$ converging to $\phi^{-1}(\widetilde{A})$ in $M$. Furthermore, the $\phi^{-1}\left(\tilde{A}_{\alpha}\right)$ are in $N$; and by the closure properties of $N$, $\phi^{-1}(\widetilde{A})$ lies in $N$. Now, applying $\phi$, we get finally that $A$ lies in $\phi(N)$, and the proof of the theorem is complete.

\subsection{Standard rings.}

Definition 3.3. Let $M$ be a semifinite ring on a Hilbert space $H$. If there exists a conjugation $J$ of $H$ such that $J M J=M^{\prime}$ and such that for $A$ in the center of $M, J A J=A^{*}$, then $M$ is said to be a standard ring.

LEMMA 3.4.1. If $M$ is a finite ring on the Hilbert space $H$, then $M$ is standard if and only if the coupling operator is the identity.

Proof. First, assume that the coupling operator is the identity operator. By Lemmas 1.2.7 and 1.2.8, there exists a family $\left\{P_{\alpha}\right\}, \alpha \in \Gamma$, of orthogonal projections in the center of $M$ such that $\sum_{\alpha \in \Gamma} P_{\alpha}=I$ and with vectors $x_{\alpha}, \alpha \in \Gamma$, such that either $\left[M x_{\alpha}\right]=P_{\alpha} H$, or $\left[M^{\prime} x_{\alpha}\right]=P_{\alpha} H$. However, since $I$ is the coupling operator, we always get both: $\left[M^{\prime} x_{\alpha}\right]=P_{\alpha} H=\left[M x_{\alpha}\right]$. But now we can apply Lemma 1.2.2 and get conjugations $J_{\alpha}, \alpha \in \Gamma$, on $P_{\alpha} H$, such that $M P_{\alpha}$ is standard with respect to $J_{\alpha}$. That $\sum_{\alpha \in \Gamma} J_{\alpha}$ is the conjugation of our lemma now follows immediately.

Next, assume that $M$ is standard. As above, pick a family $\left\{P_{\alpha}\right\}, \alpha \in \Gamma$, of orthogonal projections in the center of $M$ such that $\sum_{\alpha \in \Gamma} P_{\alpha}=I$ and such that either $\left[M x_{\alpha}\right]=P_{\alpha} H$ or $\left[M^{\prime} x_{\alpha}\right]=P_{\alpha} H$. Suppose $\left[M x_{\alpha}\right]=P_{\alpha} H$, and consider $\left[M^{\prime} x_{\alpha}\right]=\left[J M J x_{\alpha}\right]=J\left[M J x_{\alpha}\right]$. By the proof of Lemma 1.2.3, $\left[M J x_{\alpha}\right]$ $\sim P_{\alpha} H$ or $\left[M J x_{\alpha}\right]=P_{\alpha} H$. But then $\left[M^{\prime} x_{\alpha}\right]=J P_{\alpha} H=P_{\alpha} J H=P_{\alpha} H$, or $\left[M^{\prime} x_{\alpha}\right]=P_{\alpha} H$. A similar computation for the case $\left[M^{\prime} x_{\alpha}\right]=P_{\alpha} H$ shows that for all $\alpha \in \Gamma,\left[M x_{\alpha}\right]=P_{\alpha} H=\left[M^{\prime} x_{\alpha}\right]$. It is now clear that the identity operator is our coupling operator and the proof of the lemma is complete. 
Lemma 3.4.2. If $M$ is a purely infinite ring of type $S_{\Omega}$ on the Hilbert space $H$, with coupling operator $C$, then $M$ is standard if and only if $C=(\Omega / \Omega) I$.

Proof. Let $C=(\Omega / \Omega) I$. As $M$ is of type $S_{\Omega}$, there exists a family $\left\{E_{\alpha}\right\}$, $\alpha \in \Gamma$, of orthogonal, equivalent, finite projections in $M$ such that $\sum_{\alpha \in \Gamma} E_{\alpha}$ $=I$. Let $\alpha_{0}$ be a fixed element of $\Gamma$, and pick partial isometries $\left\{V_{\alpha}\right\}, \alpha \in \Gamma$, in $M$ such that $V_{\alpha}^{*} V_{\alpha}=E_{\alpha}, V_{\alpha} V_{\alpha}^{*}=E_{\alpha_{0}}$.

Notice that the mapping $A^{\prime} \rightarrow A^{\prime} E_{\alpha_{0}}$ is an isomorphism of $M^{\prime}$ onto $M_{E_{\alpha_{0}} H}^{\prime}$ since if $A^{\prime} E_{\alpha_{0}}=0$, then $A^{\prime} E_{\alpha}=0$ for all $\alpha$ with finally $A^{\prime}=0$. Thus we get $M_{E_{\alpha_{0} H}}$ finite with $M_{E_{\alpha_{0} H}}^{\prime}$ of type $S_{\Omega}$. Now, Lemma 3.2.4 gives us a family $\left\{E_{\alpha_{0}} E_{\gamma}^{\prime}\right\}, \gamma \in \Gamma$, of orthogonal, equivalent, finite projections in $M_{E_{\alpha_{0} H}}^{\prime}$ such

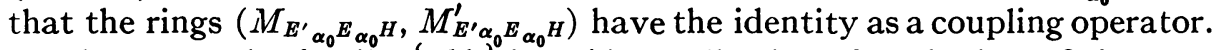
Furthermore, the family $\left\{E_{\alpha}^{\prime}\right\}^{0}$ in $M^{\prime}$ is a collection of equivalent, finite projections; and since for $\alpha \neq \alpha^{\prime}, 0=E_{\alpha}^{\prime} E_{\alpha_{0}} E_{\alpha^{\prime}}^{\prime}$, it follows that $E_{\alpha}^{\prime} E_{\alpha^{\prime}}^{\prime}=0$ or that the above collection is orthogonal. In a similar fashion, $\sum_{\alpha \in \Gamma} E_{\alpha}^{\prime}=I$.

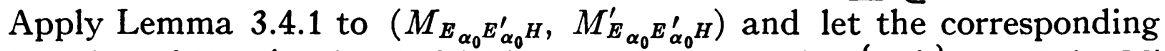
conjugation of $E_{\alpha_{0}} E_{\alpha_{0}}^{\prime} H$ be $J_{0}$. Picking partial isometries $\left\{V_{\alpha}^{\prime}\right\}, \alpha \in \Gamma$, in $M^{\prime}$ such that $V_{\alpha}^{\prime *} V_{\alpha}^{\prime}=E_{\alpha}^{\prime}, V_{\alpha}^{\prime} V_{\alpha}^{\prime *}=E_{\alpha_{0}}^{\prime}$, we define $J_{\alpha, \beta}=V_{\alpha}^{\prime *} V_{\beta}^{*} J_{0} V_{\alpha} V_{\beta}^{\prime} . J_{\alpha, \beta}$ is clearly a norm preserving mapping of $E_{\alpha} E_{\beta}^{\prime}$ onto $E_{\alpha}^{\prime} E_{\beta}$ acting like a conjugation from $E_{\alpha} E_{\beta}^{\prime}$ to $E_{\alpha}^{\prime} E_{\beta}$. Since $\sum_{\alpha, \beta \in \Gamma} E_{\alpha} E_{\beta}^{\prime}=\left(\sum_{\alpha \in \Gamma} E_{\alpha}\right)\left(\sum_{\beta \in \Gamma} E_{\beta}^{\prime}\right)$ $=I$, we can form $J=\sum_{\alpha, \beta \in \Gamma} J_{\alpha, \beta}$.

Now for any finite subsets $\Gamma^{\prime}, \Gamma^{\prime \prime}$ of $\Gamma$, we have:

$$
\begin{aligned}
J\left(\sum_{\alpha \in \Gamma^{\prime \prime}, \beta \in \Gamma^{\prime}}\right. & \left.E_{\alpha} E_{\beta}^{\prime}\right) A\left(\sum_{\delta \in \Gamma^{\prime}, \beta \in \Gamma^{\prime \prime}} E_{\gamma} E_{\delta}^{\prime}\right) J \\
& =\sum_{\alpha, \gamma \in \Gamma^{\prime \prime}, \beta, \delta \in \Gamma^{\prime}} J_{\alpha, \beta} A J_{\delta, \gamma}=\sum V_{\alpha}^{\prime *} V_{\beta}^{*} J_{0} V_{\alpha} V_{\beta}^{\prime} A V_{\delta}^{\prime *} V_{\gamma}^{*} J_{0} V_{\delta} V_{\gamma}^{\prime} \\
& =\sum_{\beta \in \Gamma^{\prime}, \alpha, \gamma \in \Gamma^{\prime \prime}} V_{\alpha}^{\prime *} V_{\beta}^{*} J_{0} E_{\alpha_{0}^{\prime}}^{\prime}\left(V_{\alpha} A V_{\gamma}^{*}\right) J_{0} V_{\beta} V_{\gamma}^{\prime}
\end{aligned}
$$

since $V_{\beta}^{\prime} V_{\delta}^{\prime *}=0$ for $\beta \neq \delta$ and equals $E_{\alpha_{0}}^{\prime}$ for $\beta=\delta$. Also, since $J_{0} E_{\alpha_{0}}^{\prime}\left(V_{\alpha} A V_{\gamma}^{*}\right) J_{0}$ lies in $M_{E_{\alpha_{0}} E_{\alpha_{0}}^{\prime}}^{\prime}$, represent it by the operator $B_{\alpha_{, \gamma}}^{\prime} E_{\alpha_{0}}$ for $B_{\alpha_{, \gamma}}^{\prime}$ in $M_{E_{\alpha_{0}}^{\prime} H}^{\prime}$. Then

$$
\begin{aligned}
J\left(\sum_{\alpha \in \Gamma^{\prime \prime}, \beta \in \Gamma^{\prime}} E_{\alpha} E_{\beta}^{\prime}(A) \sum_{\gamma \in \Gamma^{\prime \prime, \delta} \in \Gamma^{\prime}} E_{\alpha} E_{\delta}^{\prime}\right) J & \\
=\sum_{\alpha, \gamma \in \Gamma^{\prime \prime}, \beta \in \Gamma^{\prime}} V_{\alpha}^{\prime *} V_{\beta}^{*} B_{\alpha, \gamma}^{\prime} E_{\alpha_{0}} V_{\beta} V_{\gamma}^{\prime} & =\left(\sum_{\beta \in \Gamma^{\prime}} V_{\beta}^{*} V_{\beta}\right)\left(\sum_{\alpha, \gamma \in \Gamma^{\prime \prime}} V_{\alpha}^{\prime *} B_{\alpha, \gamma}^{\prime} V_{\gamma}^{\prime}\right) \\
& =\left(\sum_{\beta \in \Gamma^{\prime}} E_{\beta}\right)\left(\sum_{\alpha, \gamma \in \Gamma^{\prime \prime}} V_{\alpha}^{\prime *} B_{\alpha, \gamma}^{\prime} V_{\gamma}^{\prime}\right) .
\end{aligned}
$$

Then, by arbitrariness of $\Gamma^{\prime}$ and continuity of $J$,

$$
J\left(\sum_{\alpha \in \Gamma^{\prime \prime}} E_{\alpha}\right) A\left(\sum_{\gamma \in \Gamma^{\prime \prime}} E_{\gamma}\right) J=\sum_{\alpha, \gamma \in \Gamma^{\prime \prime}} V_{\alpha}^{\prime *} B_{\alpha, \gamma}^{\prime} V_{\gamma}^{\prime}=S_{\Gamma \prime \prime}^{\prime}
$$


in $M^{\prime}$. But the left-hand side of this equation converges to $J A J$ in the strong topology, and thus $S_{\Gamma^{\prime \prime}}^{\prime \prime}$ converges to $J A J$, proving that $J A J$ lies in $M^{\prime}$.

If $A$ lies in the center of $M$, then $B_{\alpha, \gamma}^{\prime} E_{\alpha_{0}}=J_{0} A E_{\alpha_{0}}^{\prime} V_{\alpha} V_{\gamma}^{*} J_{0}=0$ for $\alpha \neq \gamma$, $=J_{0} A E_{\alpha_{0}}^{\prime} E_{\alpha_{0}} J_{0}=A^{*} E_{\alpha_{0}}^{\prime} E_{\alpha_{0}}$ for $\alpha=\gamma$. We now get $J\left(\sum_{\alpha \in \Gamma^{\prime \prime}} E_{\alpha}\right) A\left(\sum_{\gamma \in \Gamma^{\prime \prime}} E_{\gamma}\right)$ $=\sum_{\alpha \in \Gamma^{\prime \prime}} V_{\alpha}^{\prime *} A^{*} V_{\alpha}^{\prime}=\left(\sum_{\alpha \in \Gamma^{\prime \prime}} E_{\alpha}^{\prime}\right) A^{*}$. Again by continuity of $J, J A J=A^{*}$, and $M$ is shown to be a standard ring.

Now assume that $M$ is standard of type $S_{\Omega}$. If $\left\{E_{\alpha}\right\}, \alpha \in \Gamma$, is a collection of orthogonal, equivalent, finite projections such that $\sum_{\alpha \in \Gamma} E_{\alpha}=I$, then $\sum_{\alpha \in \Gamma} J E_{\alpha} J=I$ with the family $\left\{J E_{\alpha} J\right\}, \alpha \in \Gamma$, consisting of orthogonal, equivalent, finite projections in $M^{\prime}$. Thus $M^{\prime}$ is of type $S_{\Omega}$ and the proof of the lemma is complete.

THEOREM 15. Let $M$ be a semifinite ring with coupling operator $C . M$ is standard if and only if $C$ has the form $\sum_{\alpha \in \pi}(\alpha / \alpha) P_{\alpha, \alpha}$.

Proof. Let $C=\sum_{\alpha \in \pi}(\alpha / \alpha) P_{\alpha, \alpha}$. By Lemma 3.4.1,.MP $P_{1,1}$ is standard with respect to a conjugation $J_{1}$, and by Lemma 3.4.2, the rings $M P_{\alpha}, \alpha>1$, are standard with respect to the conjugations $J_{\alpha}$. But now the conjugation $J$ $=\sum_{\alpha \in \pi} J_{\alpha}$ clearly makes $M$ a standard ring.

Let $M$ be standard with respect to $J$. If $\left\{P_{0}\right\}, \alpha \in \Gamma$, is the decomposition of $I$ into homogeneous parts given in Theorem 3, then $\left\{J P_{\alpha} J\right\}$ is clearly the decomposition of $M^{\prime}$ into homogeneous parts. But $J P_{\alpha} J=P_{\alpha}$, as the projections are in the center of $M$; and thus $P_{\alpha, \alpha^{\prime}}=P_{\alpha} \cdot P_{\alpha^{\prime}}=0$ or $P_{\alpha}$. Also, since $M P_{1}$ is standard with respect to $J, C P_{1}=P_{1}$, and $C=\sum_{\alpha \in \pi}(\alpha / \alpha) P_{\alpha, \alpha}$.

Added in proof. It will be noted that the condition "essentially bounded" occurs in several of our theorems, and it would be of interest if perhaps the condition might be removed for some. In the case of Theorem 9 , however, the following example shows that here the condition cannot be removed.

Let $M$ be the ring of all bounded operators on an infinite-dimensional Hilbert space. Now, letting $\Gamma$ consist of two elements, apply Theorem 4 to $M^{\prime}$ obtaining rings $\tilde{M}, \tilde{M}^{\prime}$ with $\tilde{M}^{*}$-isomorphic to $M$. This *-isomorphism clearly preserves the coupling operator which is not essentially finite. But, if $\phi$ could be implemented by a linear isometry $W$, then $M^{\prime}$ and $\tilde{M}^{\prime}$ would be *-isomorphic-clearly an impossibility since $M^{\prime}$ is abelian and $\tilde{M}^{\prime}$ is nonabelian.

\section{APPENDIX. WEAK AND STRONG CONTINUITY OF *-ISOMORPHISMS}

In Theorem 9 we obtained a condition for unitary equivalence, depending upon the coupling operator. In this appendix we shall obtain a condition for strong (weak) continuity of ${ }^{*}$-isomorphisms also depending upon the coupling operator.

Lemma 1. Let $M$ be a finite ring of operators on a Hilbert space $H$, with coupling operator $C$. If $\phi$ is $a^{*}$-isomorphism mapping $M$ onto a ring $\tilde{M}$ acting 
on the Hilbert space $\tilde{H}$, and if $C$ is bounded, then $\phi$ is weakly and strongly continuous.

Proof. This lemma clearly follows from Theorems 11 and 12, plus the fact that the strong (weak) topology is weaker than the strongest ( $\sigma$-weak) topology.

LEMma 2. Let $M, M^{\prime}, \tilde{M}, \tilde{M}^{\prime}$ (M finite), be semifinite rings of operators on Hilbert spaces $H, \tilde{H}$, with coupling operators $C, \tilde{C}$, respectively. If $\phi$ is $a{ }^{*}$-isomorphism mapping $M$ onto $\tilde{M}$, and if $\widetilde{C}$ is bounded, then $\phi$ is strongly (weakly) continuous if and only if $C$ is bounded.

Proof. Assume that $\phi$ is strongly (weakly) continuous. We consider the mapping $A \rightarrow A^{\natural}$ in $M$ as a composite of the mappings $A \rightarrow \phi(A) \rightarrow \phi(A)^{\natural}$ $=\phi\left(A^{\natural}\right) \rightarrow A^{\natural}$. By hypothesis, $\phi$ is continuous; and the continuity of the other maps follows from Lemma 1 , Theorem 8 , and the fact that $\widetilde{C}$ is bounded. This shows that the mapping $A \rightarrow A^{\natural}$ is continuous and finally that $C$ is bounded, by Theorem 8 .

TheOREM A. Let $M, M^{\prime}, \tilde{M}, \tilde{M}^{\prime}$ be semifinite rings of operators on Hilbert spaces $H, \tilde{H}$, with essentially bounded coupling operators $C, \tilde{C}$ respectively. Further, let $C_{0}$ and $\tilde{C}_{0}$ be the ordinary operator parts of the coupling operators, plus $\tilde{S}=$ L.U.B. $\left(\tilde{C}_{0}, \tilde{I}\right)$. Now, if $\phi$ is $a^{*}$-isomorphism mapping $M$ onto $\tilde{M}$, then $\phi$ is strongly (weakly) continuous if and only if the operator $T=C_{0} \phi^{-1}\left(\tilde{S}^{-1}\right)$ is bounded.

Proof. Let $\tilde{C}=\tilde{C}_{0}+\sum\left(1 / \alpha^{\prime}\right) \tilde{P}_{1, \alpha^{\prime}}+\sum_{\alpha>1}\left(\alpha / \alpha^{\prime}\right) \tilde{P}_{\alpha, \alpha^{\prime}}$, be the canonical form for the coupling operator, and define $\widetilde{Q}_{2}=\sum \widetilde{P}_{1, \alpha^{\prime}}, \widetilde{Q}_{3}=\sum_{\alpha>1} \tilde{P}_{\alpha, \alpha^{\prime}}$. Further, we write $\tilde{C}_{0}=\sum_{\beta \in \Gamma}\left(\tilde{C}_{\beta}+\tilde{D}_{\beta}^{-1}\right)$ as in Theorem 1. Clearly, $\tilde{S}$ $=\sum_{\beta \in \Gamma}\left(\tilde{E}_{\beta} \tilde{P}_{\beta}+\tilde{D}_{\beta}^{-1}\right)+\tilde{Q}_{2}+\tilde{Q}_{3} ;$ and thus we have for $\tilde{E}=\sum_{\beta \in \Gamma} \tilde{P}_{\left[\tilde{M}^{\prime} \tilde{x}_{\beta}\right]}$, $\tilde{Q}_{1}=\sum_{\beta \in \Gamma} \tilde{E}_{\beta} \widetilde{P}_{\beta}, \tilde{Q}_{0}=\sum_{\beta \in \Gamma} \tilde{E}_{\beta}^{\perp} \tilde{P}_{\beta}$, that $\tilde{S}^{-1}=\sum_{k=1}^{3} \tilde{Q}_{k}+\sum_{\beta \in \Gamma} \tilde{D}_{\beta}$ and hence $\tilde{S}^{-1}=\sum_{k=1}^{3} \tilde{Q}_{k}+\mathscr{E}^{4}$.

Now let $Q_{k}=\phi^{-1}\left(\tilde{Q}_{k}\right)$ and $E=\phi^{-1}(\tilde{E})$, and assume that the operator $T=C_{0} \phi^{-1}\left(\tilde{S}^{-1}\right)=C_{0}\left(E^{\natural}+Q_{1}+Q_{2}\right)$ is bounded $\left(C_{0} Q_{3}\right.$ is zero by the assumption of essential boundedness). The continuity of $\phi$ on $M Q_{3}$ follows from Lemma 3.3.2, and continuity on $M Q_{1}$ and $M Q_{2}$ is implied by Lemma 1 above. To prove the continuity of $\phi$ on $M Q_{0}$, consider the linear functional $\omega$ on $M Q_{0}$ defined by the equation $\omega(A)=(\phi(A) \tilde{x}, \tilde{x})$ for $\tilde{x}$ arbitrary in $\tilde{H}$. It is clearly a linear functional on the ring $M_{F H}$, where $\tilde{F}=\tilde{P}_{\left[\tilde{M}^{\prime} \tilde{x}\right]}, F=\phi^{-1}(\tilde{F})$, and $\tilde{F}^{\prime}=\tilde{P}_{[\widetilde{M} \tilde{x}]}$. Since $\widetilde{C}_{0} \tilde{E}^{\natural}=\tilde{Q}_{0}$, we get $\tilde{F}^{\natural}=\widetilde{F}^{\natural} \widetilde{C}_{0} \widetilde{E}^{\natural}=\widetilde{F}^{\prime \natural} \tilde{E}^{\natural} \leqq \tilde{E}^{\natural}$, and we see that $C_{0} F^{\natural}$ is dominated by the bounded operator $C_{0} E^{\natural} \leqq T$. But the operator $C_{0} F^{\natural} F$ is the coupling operator for the rings $M_{F H}, M_{F H}^{\prime}$ by an argument similar to that of the proof of Lemma 1.2.5, and thus the functional $\omega$ has the form given in Theorem 7. The continuity of $\phi$ now follows easily.

Finally, assume that $\phi$ is strongly (weakly) continuous. The boundedness 
of $C_{0}\left(Q_{1}+Q_{2}\right)$ clearly follows from Lemma 2 and so it remains to prove the boundedness of $C_{0} E^{\natural}$. But the boundedness simply follows from Lemma 2 applied to the rings $M_{E H}, M_{E H}^{\prime}, \tilde{M}_{\widetilde{E} \widetilde{H}}, \tilde{M}_{\widetilde{E} \tilde{H}}^{\prime}$ with coupling operators $C_{0} E^{\natural} E, \tilde{E}$; and the proof of the theorem is complete.

\section{LIST OF REFERENCES} 261.

1. J. Dixmier, Les anneaux d'opérateurs de classe finie, Ann. École Norm. (1949) pp. 209-

2. H. A. Dye, Radon-Nikodým theorems for operator algebras, Thesis, University of Chicago, 1950.

3. I. Kaplansky, Quelques resultats sur les anneaux d'opérateurs, C. R. Acad. Sci. Paris vol. 231, pp. 485-486.

4. - Projections in Banach algebras, Ann. of Math. vol. 53, pp. 235-249.

5. J. von Neumann and F. J. Murray, On rings of operators, Ann. of Math. vol. 37, pp. $116-229$.

6. J. von Neumann, On rings of operators, II. Trans. Amer. Math. Soc. vol. 41, pp. 208-248.

7. - On rings of operators. IV, Ann. of Math. vol. 44, pp. 716-808. 715.

8. - On some algebraic properties of operator rings, Ann. of Math. vol. 44, pp. 709-

9. I. E. Segal, Decompositions of operator algebras. II, Memoirs of the American Mathematical Society, no. 9.

University OF Chicago,

Chicago, Ill. 